\title{
The Efficient Computation of Bounds for Functionals of Finite Element Solutions in Large Strain Elasticity
}

\author{
J. Bonet ${ }^{1}$, A. Huerta ${ }^{2}$ and J. Peraire ${ }^{3}$ \\ ${ }^{1}$ Department of Civil Engineering, University of Wales Swansea, Swansea SA2 8PP, UK \\ 2 Departamento de Matematica Aplicada III, Universitat Politecnica de Catalunya, 08034 Barcelona, Spain \\ 3 Department of Aeronautics and Astronautics, Massachusetts Institute of Technology, Cambridge, MA 02139, USA
}

\begin{abstract}
We present an implicit a-posteriori finite element procedure to compute bounds for functional outputs of finite element solutions in large strain elasticity. The method proposed relies on the existence of a potential energy functional whose local minima, over a space of suitably chosen continuous functions, corresponds to the problem solution. The output of interest is cast as a constrained minimization problem over an enlarged discontinuous finite element space. A Lagrangian is formed were the multipliers are an adjoint solution, which enforces equilibrium, and hybrid fluxes, which constrain the solution to be continuous. By computing approximate values for the multipliers on a coarse mesh, strict upper and lower bounds for the output of interest on a suitably refined mesh, are obtained. This requires a minimization over a discontinuous space, which can be carried out locally at low cost. The computed bounds are uniformly valid regardless of the size of the underlying coarse discretization. The method is demonstrated with two applications involving large strain plane stress incompressible neo-hookean hyperelasticity.
\end{abstract}

\section{Introduction}

Engineering applications require the prediction of certain quantities of interest, or outputs - such as compliance, stresses, flow rates, heat transfer. These outputs are functionals of field variables - such as displacement, velocity or, temperature - which, in turn, are solutions of partial differential equations that need to be approximated numerically. Engineering decisions are thus based on approximations to the desired outputs that are generated from computed approximations to the field variables. It is often difficult to determine a-priori the size of the discretization that would yield the outputs at a required level of accuracy. In fact, it is well known that for a given field solution, different outputs can be predicted at varying levels of accuracy. The situation is further complicated in a real design, or optimization, setting where one may require multiple 
appeals to the PDE solver to evaluate solutions for different values of the design parameters. In this case, using a conservative fine discretization for every solution, in an attempt to guarantee a prescribed accuracy, may prove prohibitive.

In this paper, we consider the non-linear equations describing the large strain deformation of a hyperelastic material. We propose a finite element a-posteriori method to compute inexpensive upper and lower bounds for engineering outputs of interest. The proposed method requires the solution of non-linear local Neumann subproblems and is therefore referred to as an implicit method [2]. When compared to the simpler less expensive a-posteriori explicit methods, which only require residual evaluations (e.g. [3]), implicit methods offer the potential for quantitative constant-free bounds. The original implicit methods $[9,1,5]$ were developed, for linear self-adjoint problems, to provide bounds for the energy norm of the error. In reality however, it is not the error in the energy norm which is of interest, but the error in the quantities on which an engineering decision will be based - for instance deflection or stress. The first attempts at developing a-posteriori error estimation for functional outputs were carried out in the context of explicit error estimation $[6,16,17]$. It turns out that the error in the quantities of interest can be related to a weighted norm of the residual where the specific weights are determined by the solution of an adjoint problem. Unfortunately, the explicit nature of the procedure means that these estimates contain unknown constants, which severely limit its quantitative value.

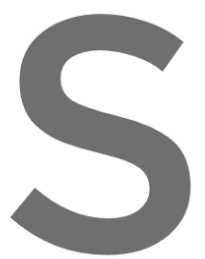

The development of an ir outputs of partial differention coercive problems includin position technique and an implicit procedure yieldi
ing non-symmetric terms.
he construction of an augm
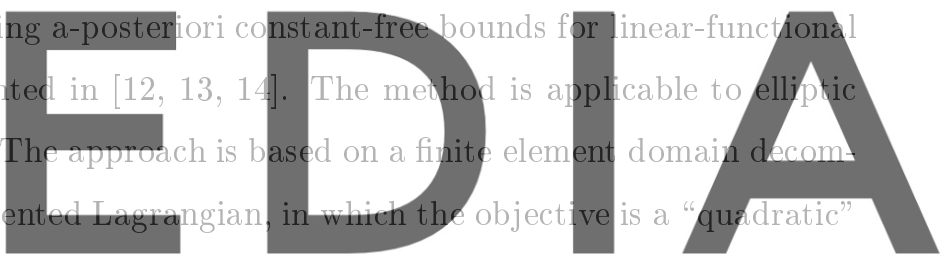

energy re-formulation of the desired output, and the constraints are the finite element equilibrium equations

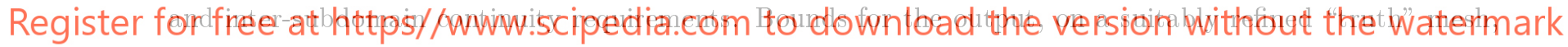

are then obtained by appealing to a dual min-max relaxation, evaluated for lagrange multipliers computed on a coarse "working" mesh. One attractive feature of this approach is its natural extension to non-linear problems and outputs. Previous a-posteriori error estimation methods for non-linear partial differential equations, are based on a linearized problem, and hence, only provide meaningful estimates once the computed solution is in the asymmptotic convergence range. The proposed approach, on the other hand, is formulated directly in the non-linear context. Although the bounds are uniformly valid, they are found to converge to the true output at the optimal rate when the asymmptotic regime is reached. To our knowledge, this is the first time that a procedure that yields a-posteriori strict bounds for outputs of engineering interest, in the context of large strain fully non-linear hyperelastic materials, has been proposed. The procedure is illustrated for a two dimensional, incompressible neo-hookean hyperelastic material under plane stress, but it is generally applicable to general three dimensional finite strain models.

The focus of this paper is on computing bounds, but the method presented provides naturally a local indicator that can be used to drive a mesh adaptive procedure as shown in [14]. 
The computation of the error for quantities of interest has been an active area of research in recent years. The separate treatment of local an pollution errors was initiated in [4]. The implicit a posteriori error estimation method described in [15], is applicable to self-adjoint problems and is closely related to the approach followed here. Finally, explicit a posteriori estimates and adaptive strategies for large strain elasticity, are developed in [10].

The paper is organized as follows. First, we introduce the model problem and formulation that will be used as vehicle to illustrate our method. We describe the necessary preliminaries and present a bound procedure for the total potential energy. Next, we develop the extension to arbitrary functional outputs. We present numerical results for two examples commonly studied in the literature, and conclude with a discussion on the advantages and drawbacks of the proposed approach.

\section{Problem Formulation}

Consider the finite deformation of a 2D body under the action of a distributed traction on its boundary as illustrated in figure 1. Let $\Omega$ represent the two dimensional domain corresponding to the undeformed configuration, and $\mathbf{X}=\left(X_{1}, X_{2}\right) \in \Omega$, denote the material coordinates of a given point. The boundary of $\Omega$ is denoted by $\Gamma=\overline{\Gamma_{D}^{1} \cup \Gamma_{N}^{1}}=\overline{\Gamma_{D}^{2} \cup \Gamma_{N}^{2}}$. Here, $\Gamma_{D}^{i}, i=1,2$ is the portion of the boundary where the motion

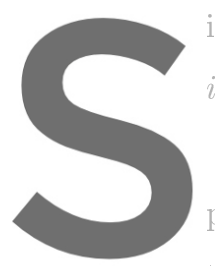

in the $i$-th direction is

i-th component of the trar

A motion is described

particle X. We define the
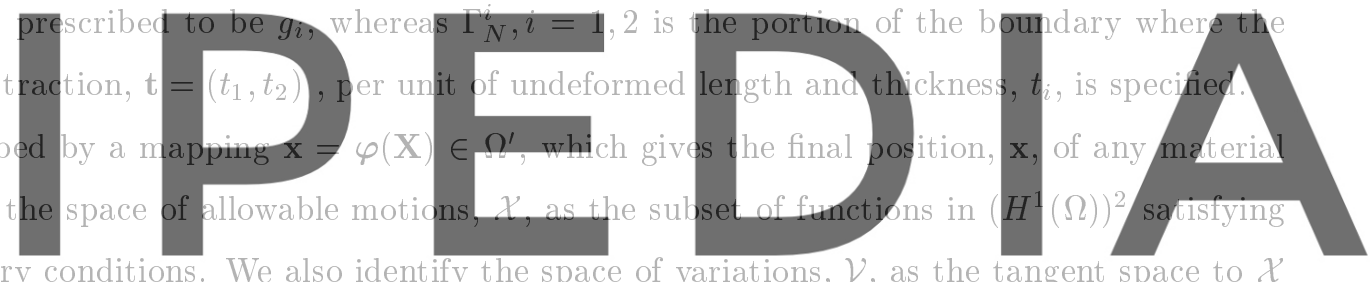

Register fdr] freensat https//www.scipedia.com to download the version without the watermark

$$
\begin{gathered}
\mathcal{X}=\left\{\varphi=\left(\varphi_{1}, \varphi_{2}\right) \in\left(H^{1}(\Omega)\right)^{2}\left|\varphi_{i}\right|_{\Gamma_{D}^{i}}=g_{i} \text { for } i=1,2\right\}, \\
\mathcal{V}=\left\{\mathbf{v}=\left(v_{1}, v_{2}\right) \in\left(H^{1}(\Omega)\right)^{2}\left|v_{i}\right|_{\Gamma_{D}^{i}}=0 \text { for } i=1,2\right\} .
\end{gathered}
$$

The body is considered to be sufficiently thin so that a constant strain can be assumed throughout its thickness. In this case, the plane stress incompressible neo-Hookean strain energy function per unit initial area, $\psi$, can be expressed, in terms of the deformation gradient tensor, $\mathbf{F}=\partial \varphi / \partial \mathbf{X}$, as [7]:

$$
\psi(\mathbf{F})=\frac{1}{2} \mu H\left(\mathbf{F}: \mathbf{F}+(\operatorname{det} \mathbf{F})^{-2}-3\right),
$$

where $\mu$, is the initial shear modulus and $H$, the initial thickness.

Finally, the total energy potential, $\Pi: \mathcal{X} \rightarrow \mathbb{R}$, is given by,

$$
\Pi(\varphi)=\int_{\Omega} \psi(\mathbf{F}) d \Omega-\sum_{i=1}^{2} \int_{\Gamma_{N}^{i}} t_{i} \varphi_{i} H d S .
$$




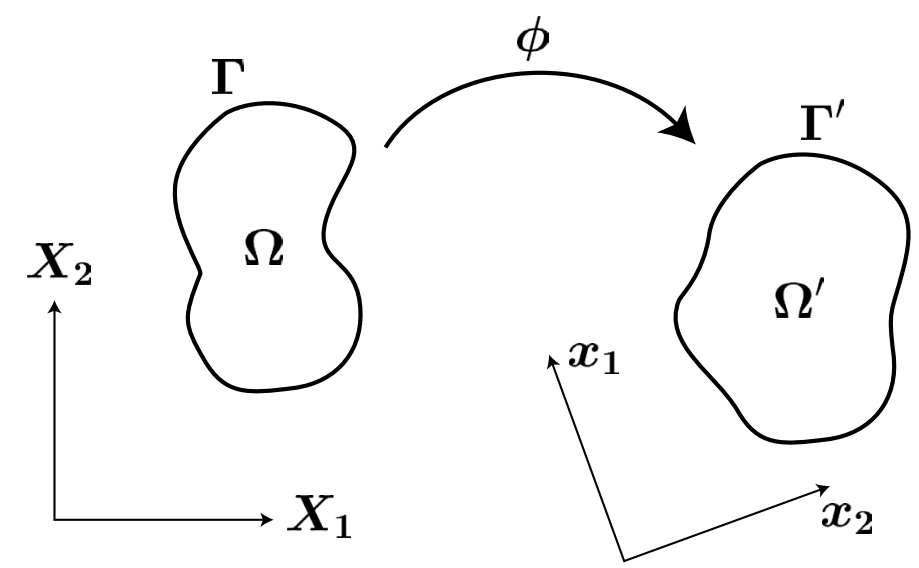

Figure 1: 2D body in plane stress

\subsection{Minimization Statement}

The equilibrium configuration is given by the motion $\phi \in \mathcal{X}$ that minimizes the total potential energy,

$$
\Pi(\phi)=\inf _{\varphi \in \mathcal{X}} \Pi(\varphi) .
$$

We note that, in general, $\Pi$ will not be convex and may have either none or multiple minima. This issue

will be further discussed below, but, for current purposes, we shall assume that $\Pi$ has a unique minimum.
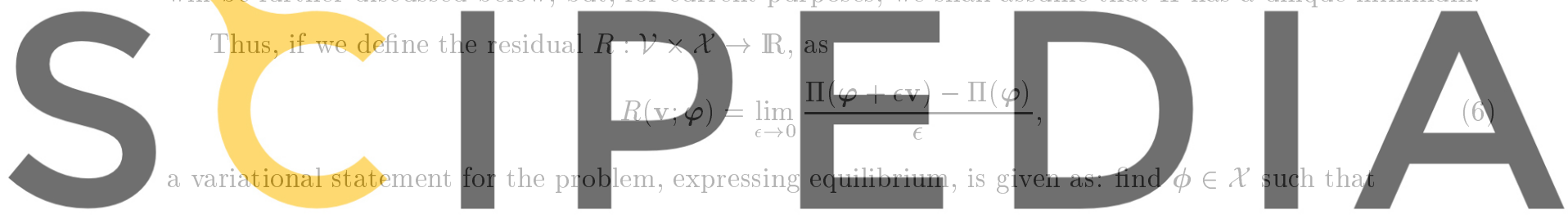

$R(\mathrm{v} ; \phi)=0$,

(7)

Register for free at https//www.scipedia.com to download the version without the watermark

In most situations, we are not ultimately interested in $\phi$, but in specific outputs that depend on $\phi$. Such

are, for instance, the motion of a point, or the average stress over a certain portion of the body. To this end, we introduce the output functional $\mathcal{S}(\varphi): \mathcal{X} \rightarrow \mathbb{R}$, and express our output of interest, $s$, as

$$
s=\mathcal{S}(\phi)
$$

In principle, $\mathcal{S}$ can be a non-linear functional as discussed later, although at present we have only applied the proposed technology to linear outputs.

We define, for future use the tangent form $K:(\mathcal{V})^{2} \times \mathcal{X} \rightarrow \mathbb{R}$, as

$$
K\left(\mathbf{v}_{1}, \mathbf{v}_{2} ; \varphi\right)=\lim _{\epsilon \rightarrow 0} \frac{R\left(\mathbf{v}_{2} ; \varphi+\epsilon \mathbf{v}_{1}\right)-R\left(\mathbf{v}_{2} ; \varphi\right)}{\epsilon},
$$

and the Hessian form $D K:(\mathcal{V})^{3} \times \mathcal{X} \rightarrow \mathbb{R}$, as

$$
D K\left(\mathbf{v}_{1}, \mathbf{v}_{2}, \mathbf{v}_{3} ; \varphi\right)=\lim _{\epsilon \rightarrow 0} \frac{K\left(\mathbf{v}_{2}, \mathbf{v}_{3} ; \varphi+\epsilon \mathbf{v}_{1}\right)-K\left(\mathbf{v}_{2}, \mathbf{v}_{3} ; \varphi\right)}{\epsilon} .
$$

We point out that, by construction, the form $R$ is linear with respect to its first argument; and $K$ and $D K$ are linear and symmetric with respect to their two, and three, first arguments, respectively. 


\section{$3 \quad$ Finite Element Spaces}

We consider two triangulations of the computational domain $\Omega$ : a working or design $H$-mesh, $\mathcal{T}_{H}$, consisting of $n_{H}$ elements, $T_{H}$; and the fine $h$-mesh, $\mathcal{T}_{h}$, consisting of $n_{h}$ elements $T_{h}$. We assume that $\mathcal{T}_{h}$ can be obtained by uniform refinement of $\mathcal{T}_{H}$. To each of these meshes we associate piecewise linear continuous finite element subpaces,

$$
\begin{aligned}
\mathcal{X}_{H} & =\left\{\varphi \in \mathcal{X}|\varphi|_{T_{H}} \in\left(\mathbf{P}_{\mathbf{1}}\left(T_{H}\right)\right)^{2}, \forall T_{H} \in \mathcal{T}_{H}\right\}, \\
\mathcal{X}_{h} & =\left\{\varphi \in \mathcal{X}|\varphi|_{T_{h}} \in\left(\mathbf{P}_{\mathbf{1}}\left(T_{h}\right)\right)^{2}, \forall T_{h} \in \mathcal{T}_{h}\right\},
\end{aligned}
$$

where $\mathbf{P}_{1}(T)$ denotes the space of linear polynomials over $T$. We have that, by construction, $\mathcal{X}_{H} \subset \mathcal{X}_{h} \subset \mathcal{X}$.

The algorithms to be presented require that our forms be expressed as sums of contributions over $\mathrm{H}$ elements $T_{H}$. Towards this end, we introduce the "broken" spaces $\hat{\mathcal{X}}_{H}$ and $\hat{\mathcal{X}}_{h}$,

$$
\begin{aligned}
& \hat{\mathcal{X}}_{H}=\left\{\varphi \in L_{2}(\Omega)|\varphi|_{T_{H}} \in\left(\mathbb{P}_{1}\left(T_{H}\right)\right)^{2}, \forall T_{H} \in \mathcal{T}_{H}\right\}, \\
& \hat{\mathcal{X}}_{h}=\left\{\varphi \in L_{2}(\Omega)|\varphi|_{T_{H}} \in \mathbb{Z}_{h}\left(T_{H}\right), \forall T_{H} \in \mathcal{T}_{H}\right\} .
\end{aligned}
$$

Here, $L_{2}(\Omega)$ is the space of square-integrable functions over $\Omega$, and $\mathcal{Z}_{h}\left(T_{H}\right)$ is defined as

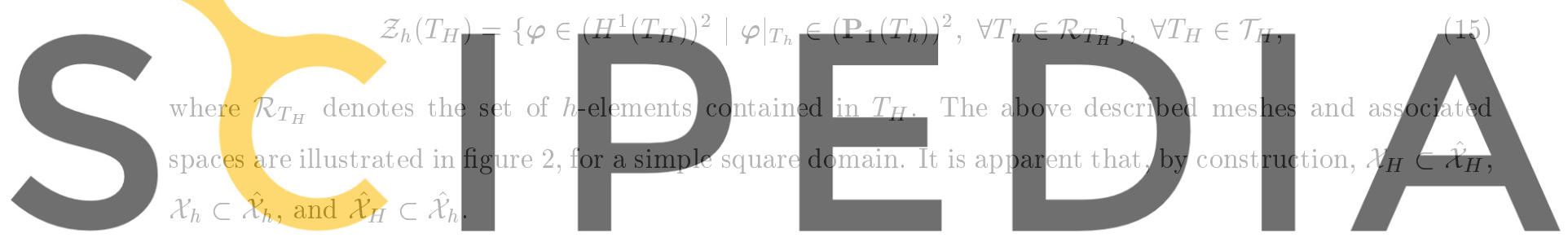

We can also define the tangent finite element spaces $\mathcal{V}_{H}, \mathcal{V}_{h}, \hat{V}_{H}$ and $\hat{V}_{h}$ in an analogous manner.

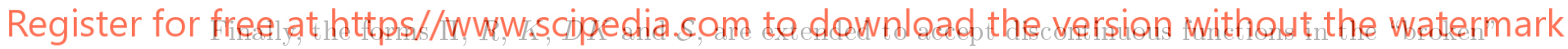

spaces by redefining these forms as a sum of $H$-element contributions. For instance, $K$ is now written as

$$
K\left(\mathbf{v}_{1}, \mathbf{v}_{2} ; \varphi\right)=\sum_{T_{H} \in \mathcal{T}_{H}} K_{T_{H}}\left(\left.\mathbf{v}_{1}\right|_{T_{H}},\left.\mathbf{v}_{2}\right|_{T_{H}} ;\left.\varphi\right|_{T_{H}}\right) ;
$$

with similar expressions for $\Pi, R, D K$ and $\mathcal{S}$.

\subsection{Continuity Form}

Let $\mathcal{E}\left(\mathcal{T}_{H}\right)$ (respectively, $\mathcal{E}\left(\mathcal{T}_{h}\right)$ ) denote the set of open edges in the triangulation $\mathcal{T}_{H}$ (respectively, $\mathcal{T}_{h}$ ). We introduce a space of functions over the element edges $\gamma_{H} \in \mathcal{E}\left(\mathcal{T}_{H}\right)$,

$$
\mathcal{Q}_{H}=\left\{\mathbf{q}=\left.\left(q_{1}, q_{2}\right)\right|_{\gamma_{H}} \in\left(\mathbf{P}_{1}\left(\gamma_{H}\right)\right)^{2}, \forall \gamma_{H} \in \mathcal{E}\left(\mathcal{T}_{H}\right)\left|q_{1}\right|_{\Gamma_{N}^{1}}=0,\left.q_{2}\right|_{\Gamma_{N}^{2}}=0\right\}
$$

analogously, for edges $\gamma_{h} \in \mathcal{E}\left(\mathcal{T}_{h}\right) \cap \mathcal{E}\left(\mathcal{T}_{H}\right)$,

$$
\mathcal{Q}_{h}=\left\{\mathbf{q}=\left.\left(q_{1}, q_{2}\right)\right|_{\gamma_{h}} \in\left(\mathbf{P}_{1}\left(\gamma_{h}\right)\right)^{2}, \forall \gamma_{h} \in \mathcal{E}\left(\mathcal{T}_{h}\right) \cap \mathcal{E}\left(\mathcal{T}_{H}\right)\left|q_{1}\right|_{\Gamma_{N}^{1}}=0,\left.q_{2}\right|_{\Gamma_{N}^{2}}=0\right\}
$$




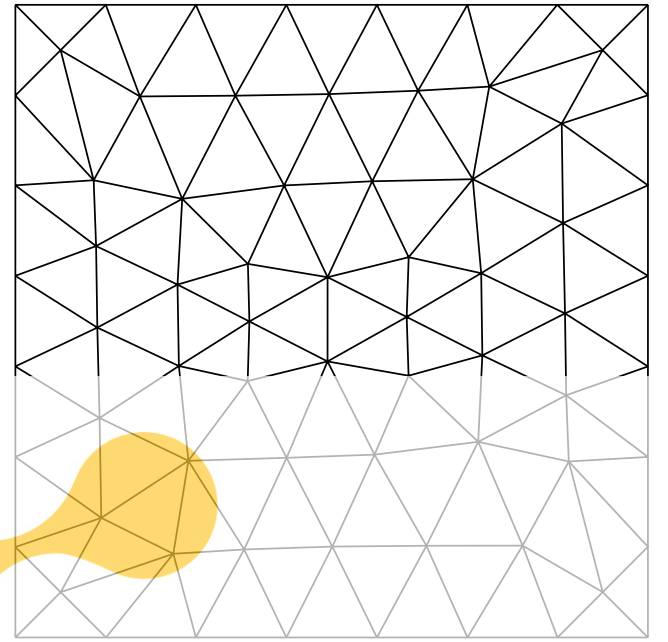

$\mathcal{X}_{H}\left(\mathcal{V}_{H}\right)$
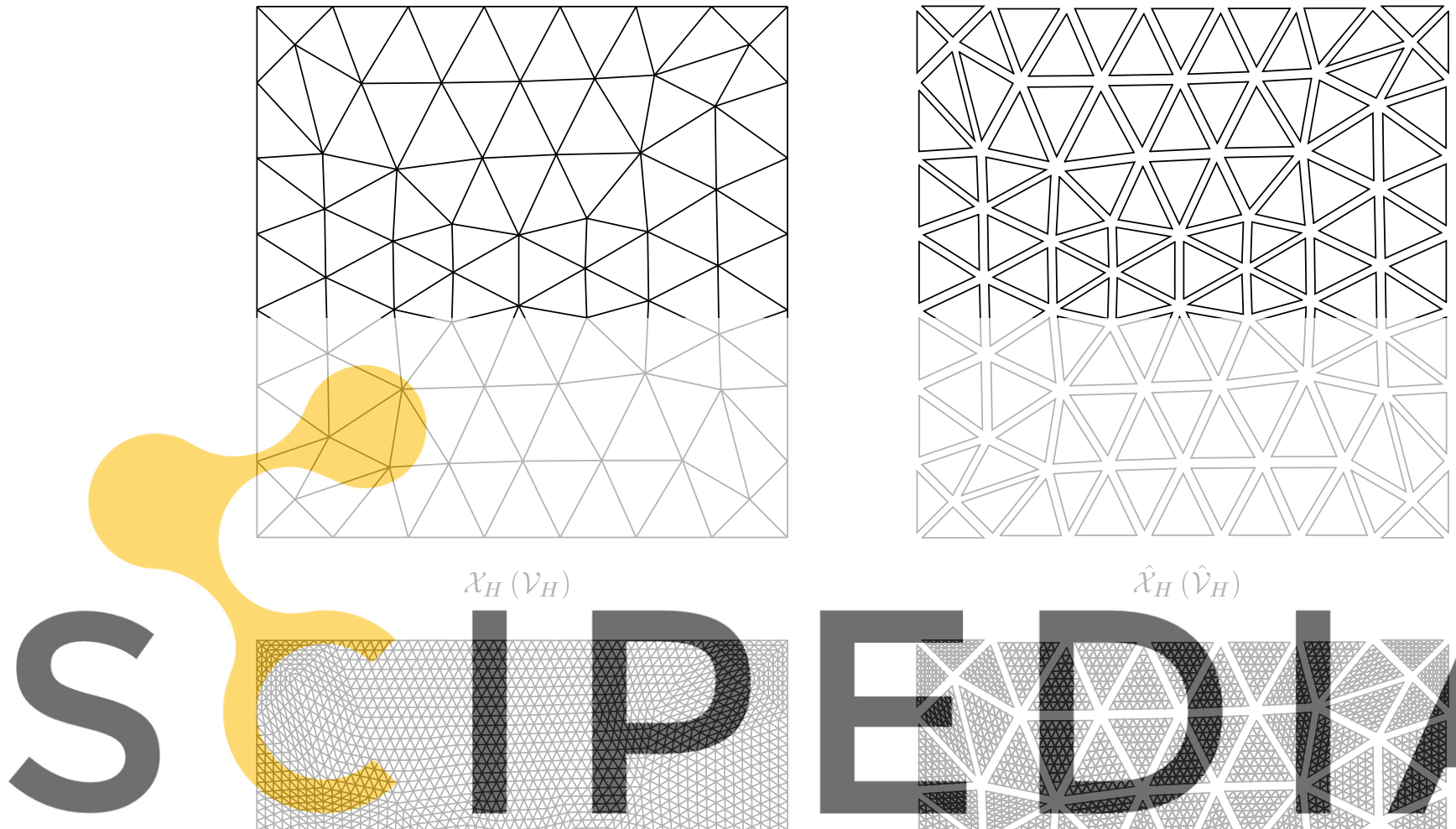

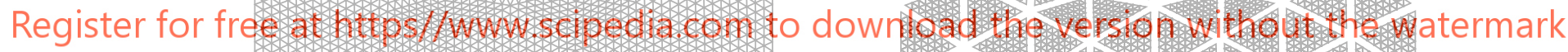

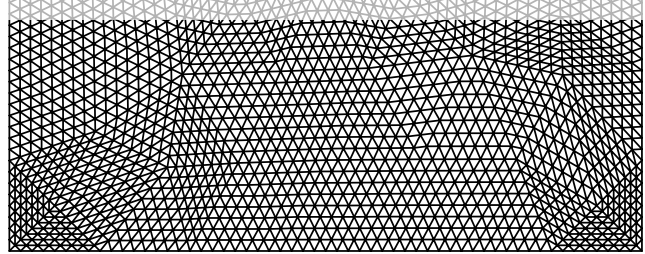

$\mathcal{X}_{h}\left(\mathcal{V}_{h}\right)$

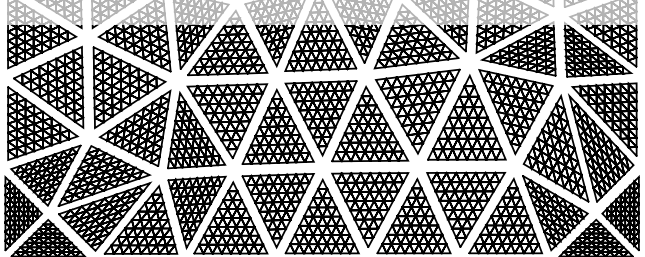

$\hat{\mathcal{X}}_{h}\left(\hat{\mathcal{V}}_{h}\right)$

Figure 2: Illustration of $\mathcal{X}_{H}\left(\mathcal{V}_{H}\right), \mathcal{X}_{h}\left(\mathcal{V}_{h}\right), \hat{\mathcal{X}}_{H}\left(\hat{\mathcal{V}}_{H}\right), \hat{\mathcal{X}}_{h}\left(\hat{\mathcal{V}}_{h}\right)$. 
It follows that $\mathcal{Q}_{H} \subset \mathcal{Q}_{h} \subset L_{2}\left(\mathcal{E}\left(\mathcal{T}_{H}\right)\right)$, and the functions in these spaces can, of course, be discountinuous.

Next, we introduce the "jump" bilinear form $b: \hat{\mathcal{X}} \times \mathcal{Q}_{h} \rightarrow \mathbb{R}$.

$$
b(\boldsymbol{\varphi}, \mathbf{q})=\left.\sum_{\gamma_{H} \in \mathcal{E}\left(\mathcal{T}_{H}\right)} \int_{\gamma_{H}}[\varphi]_{\gamma_{H}} \cdot \mathbf{q}\right|_{\gamma_{H}} H d S
$$

where $[\boldsymbol{\varphi}]_{\gamma_{H}}$, is the jump in $\varphi$ across $\gamma_{H}$, when $\gamma_{H}$ is an interior edge, and takes the value of $\varphi$ on $\gamma_{H}$, when $\gamma_{H}$ is on the boundary $\Gamma$. Interior edges are given an arbitrary orientation so that the sign of $[\varphi]_{\gamma_{H}}$ is uniquely defined. The form (19), can be used to enforce continuity on functions in $\hat{\mathcal{X}}_{H}$ and $\hat{\mathcal{X}}_{h}$; in particular,

$$
\begin{aligned}
\mathcal{X}_{H} & \equiv\left\{\varphi \in \hat{\mathcal{X}}_{H} \mid b(\boldsymbol{\varphi}, \mathbf{q})=0, \forall \mathbf{q} \in \mathcal{Q}_{H}\right\} \\
\mathcal{X}_{h} & \equiv\left\{\boldsymbol{\varphi} \in \hat{\mathcal{X}}_{h} \mid b(\boldsymbol{\varphi}, \mathbf{q})=0, \forall \mathbf{q} \in \mathcal{Q}_{h}\right\}
\end{aligned}
$$

We note that the form $b(\cdot, \cdot)$ places no restriction on $\varphi$ on natural boundaries.

\section{Bound Procedure for the Total Potential Energy}

\subsection{The coarse mesh problem}

The coarse mesh solution $\phi_{H}$ is obtained by considering the minimization statement (5) over the functions

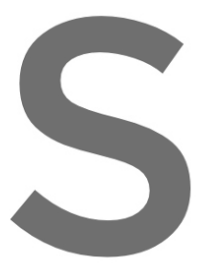

in $\mathcal{X}_{H}$. If $\Pi_{H}$, denotes
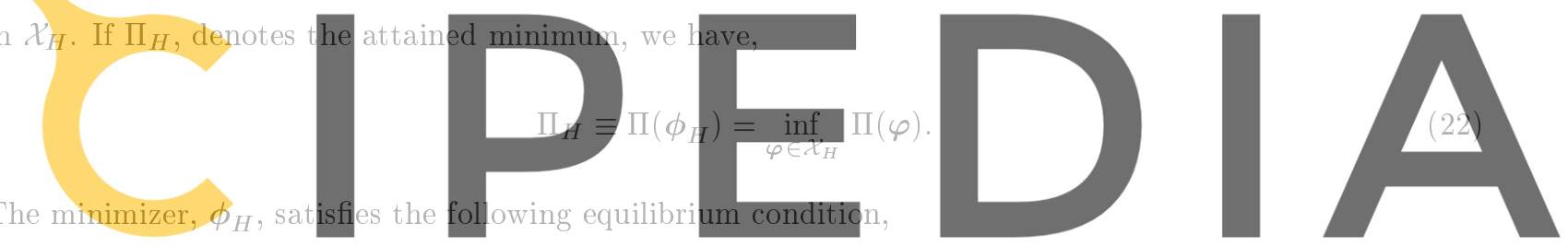

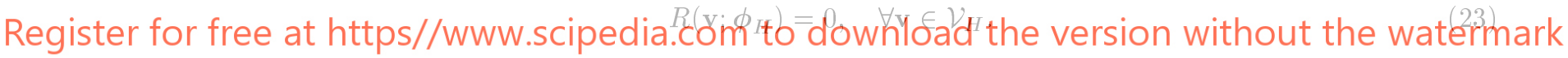

This statement represents a set of nonlinear coupled algebraic equations which can be conveniently solved using an iterative Newton-Raphson procedure [7]. More specifically, the following recursive expression can be use to determine the $i+1$-th iterate, $\phi_{H}^{i+1}$, given $\phi_{H}^{i}$ : find $\phi_{H}^{i+1} \in \mathcal{X}_{H}$ such that

$$
K\left(\mathbf{v}, \phi_{H}^{i+1}-\phi_{H}^{i} ; \phi_{H}^{i}\right)=-R\left(\mathbf{v} ; \phi_{H}^{i}\right), \quad \forall \mathbf{v} \in \mathcal{V}_{H}
$$

A fine mesh solution $\phi_{h}$, corresponding to the minimizer of (5) over $\mathcal{X}_{h}$,

$$
\Pi_{h} \equiv \Pi\left(\phi_{h}\right)=\inf _{\varphi \in \mathcal{X}_{h}} \Pi(\varphi)
$$

could, in principle, be computed in an analogous manner. Since $\mathcal{X}_{H} \in \mathcal{X}_{h}$, it follows that $\Pi_{H}$ is automatically an upper bound for the total potential energy at $\phi_{h}$, $\Pi_{h}$; i.e. $\Pi_{h} \leq \Pi_{H} \equiv \Pi_{h}^{+}$.

For linear elements, we observe the $H^{1}$-norm of the solution error to be $\mathcal{O}(H)$ whenever the solution is sufficiently regular, that is ||$\phi-\phi_{H} \|_{1} \sim \mathcal{O}(H)$, and $\left|\Pi-\Pi_{H}\right| \sim \mathcal{O}\left(H^{2}\right)$. Hence, if we assume $h$ sufficiently small so that $\phi_{h} \sim \phi$, then we have $\Pi_{h}^{+}-\Pi \sim \mathcal{O}\left(H^{2}\right)$. 


\subsection{Lagrangian formulation}

Our objective now is to compute a lower bound for $\Pi_{h}$, without requiring the solution of an expensive fine mesh problem. In principle, a lower bound for $\Pi_{h}$, will be obtained whenever the minimization in (5) is done over a space which contains $\mathcal{X}_{h}$. We recall that one such space would be $\hat{\mathcal{X}}_{h}$. Unfortunately, direct minimization over $\hat{\mathcal{X}}_{h}$ would lead to a larger problem than that of calculating $\Pi_{h}$ exactly, and worst yet, lead to a minimum which, in general, would be $-\infty$.

In order to overcome this difficulty we proceed as follows. Given (21), we can rewrite (25) as a constrained minimization problem over $\hat{\mathcal{X}}_{h}$,

$$
\Pi_{h}=\inf _{\varphi \in \mathcal{X}_{h}} \Pi(\varphi)=\left\{\begin{array}{l}
\inf _{\varphi \in \hat{\mathcal{X}}_{h}} \\
b(\boldsymbol{\varphi}, \mathbf{q})=0, \forall \mathbf{q} \in \mathcal{Q}_{h}
\end{array}\right.
$$

An augmented Lagrangian $\mathcal{L}: \hat{\mathcal{X}} \times \mathcal{Q}_{h} \rightarrow \mathbb{R}$, can now be defined as

$$
\mathcal{L}(\varphi, \mathbf{q})=\Pi(\varphi)+b(\varphi, \mathbf{q})
$$

where $\mathbf{q}$, plays the role of a lagrange multiplier and will be referred to as a hybrid flux [8]. The solution of the constrained minimization problem (26), $\phi_{h} \in \mathcal{X}_{h}$, and $\mathrm{p}_{h} \in \mathcal{Q}_{h}$, can thus be expressed as a saddle point
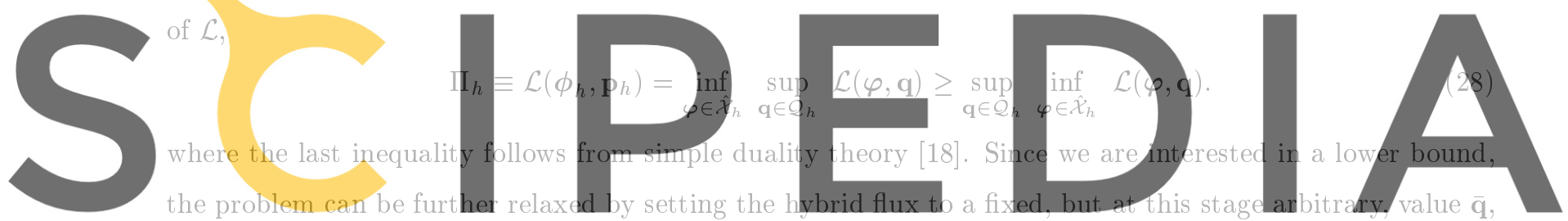

thus,

Register for free at https//www.scipedia.com, to downl oad the version without the watermark

which defines the lower bound $\bar{\Pi}_{h}^{-}(\overline{\mathbf{q}})$ as:

$$
\Pi_{h}^{-}(\overline{\mathbf{q}}) \equiv \mathcal{L}\left(\hat{\phi}_{h}, \overline{\mathbf{q}}\right)=\inf _{\varphi \in \hat{\mathcal{X}}_{h}} \mathcal{L}(\varphi, \overline{\mathbf{q}})
$$

The important point here is that, whereas the problem (28) is large and expensive to solve, the minimization (29) is carried out over the "broken" space $\hat{\mathcal{X}}_{h}$ and therefore can be solved for each $H$-macroelement in a decoupled manner as shown in the Appendix I.

The accuracy of the lower bound will depend on the duality gap in (28), and on the choice of hybrid fluxes $\overline{\mathbf{q}}$. In principle, we expect the duality gap to be zero provided that sufficient regularity conditions are met. An inexpensive choice of hybrid fluxes which is based on the coarse grid solution $\phi_{H}$, and yields optimal bound convergence (i.e. $\left.\Pi_{h}-\Pi_{h}^{-} \sim \mathcal{O}\left(H^{2}\right)\right)$ is presented below. 


\subsection{Hybrid Fluxes}

In order to determine suitable approximations to the hybrid fluxes $\overline{\mathbf{q}}$, we look for the saddlepoint of the Lagrangian (27), in the coarse grid subspaces $\hat{\mathcal{X}}_{H} \subset \hat{\mathcal{X}}_{h}$ and $\mathcal{Q}_{H} \subset \mathcal{Q}_{h}$. In particular, we look for $\phi_{H}$ and $\mathbf{p}_{H}$ such that

$$
\Pi_{H} \equiv \mathcal{L}\left(\phi_{H}, \mathbf{p}_{H}\right)=\inf _{\varphi \in \hat{\mathcal{X}}_{H}} \sup _{\mathbf{q} \in \mathcal{Q}_{H}} \mathcal{L}(\boldsymbol{\varphi}, \mathbf{q}),
$$

and then set $\overline{\mathbf{q}}=\mathbf{p}_{H}$. It is now clear that, the solution $\phi_{H}$ to the above problem can be obtained directly by solving the coarse grid problem (22) over $\mathcal{X}_{H}$. Once $\phi_{H}$ is known, the hybrid fluxes can be determined. First, we note that stationary point of the Lagrangian, will satisfy

$$
R\left(\mathbf{v} ; \phi_{H}\right)+b\left(\mathbf{v}, \mathbf{p}_{H}\right)=0, \quad \forall \mathbf{v} \in \hat{\mathcal{X}}_{H}
$$

The above equation represents a solvable but indeterminate system. The solution of this problem is known as the equilibration problem. From the physical point of view, these hybrid fluxes represent tractions that must be applied so that the each H-macroelement is in equilibrium when considered in isolation. We follow here the approach proposed in $[9,5,1]$, which requires solving an indeterminate system at each vertex of the $\mathcal{T}_{H}$ grid, the size of which is given by the number of edges that meet at the vertex.

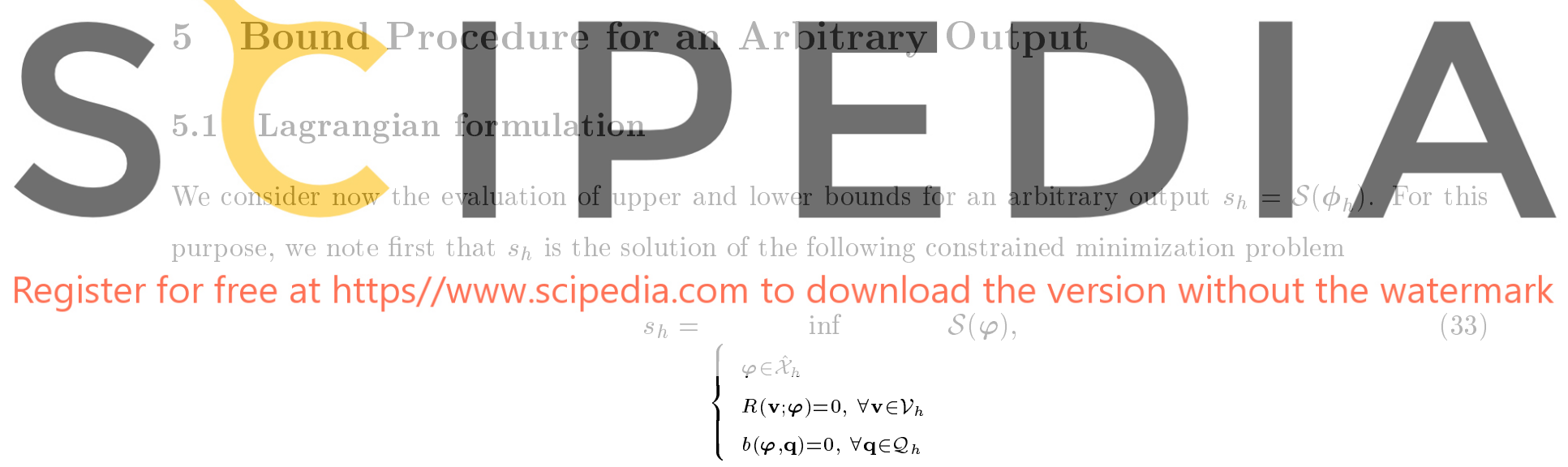

where the chosen constraints simply force $\varphi$, to be continuous and satisfy equilibrium thus being equal to $\phi_{h}$ and hence, $\mathcal{S}(\varphi)$ becomes $\mathcal{S}\left(\phi_{h}\right)$. It is now possible to re-write the above constrained minimization problem as a saddle point problem as

$$
\gamma s_{h}=\inf _{\boldsymbol{\varphi} \in \hat{\mathcal{X}}_{h}} \sup _{\substack{\mathbf{v} \in \mathcal{V}_{h} \\ \mathbf{q} \in \mathcal{Q}_{h}}} \mathcal{L}_{h}^{\gamma}(\boldsymbol{\varphi}, \mathbf{v}, \mathbf{q})
$$

where the Lagrangian functional is now

$$
\mathcal{L}_{h}^{\gamma}(\boldsymbol{\varphi}, \mathbf{v}, \mathbf{q})=\Pi(\varphi)-\Pi\left(\phi_{h}\right)+\gamma \mathcal{S}(\varphi)+R(\mathbf{v} ; \varphi)+b(\varphi, \mathbf{q}), \quad \forall \gamma \in \mathbb{R}^{+}
$$

Note that the first two terms in the above expression will cancel each other when $\varphi=\phi_{h}$. In addition, the positive factor $\gamma$ in the above equation, although arbitrary at this stage, has the function of scaling the 
physical dimensions of the output $s$ to equal those of energy, thus matching other terms in the expression for $\mathcal{L}_{h}^{\gamma}$. Finally, the last two terms in equation (35) contain the Lagrange multipliers, $\mathbf{v}$ and $\mathbf{q}$, known as the adjoint and hybrid fluxes respectively, which enforce the required constraints.

Duality and a relaxation of the constraints, by choosing fixed but arbitrary adjoint, $\mathbf{v}=\overline{\mathbf{v}}$, and hybrid fluxes, $\mathbf{q}=\overline{\mathbf{q}}$, in equation (34), gives a lower bound expression for the output $s_{h}$ as

$$
s_{h} \geq \frac{1}{\gamma} \inf _{\varphi \in \hat{\mathcal{X}}_{h}} \mathcal{L}_{h}^{\gamma}(\varphi, \overline{\mathbf{v}}, \overline{\mathbf{q}}), \quad \forall \overline{\mathbf{q}} \in \mathcal{Q}, \quad \forall \overline{\mathbf{v}} \in \mathcal{V}_{h}, \quad \forall \gamma \in \mathbb{R}^{+}
$$

The presence of the unknown potential $\Pi\left(\phi_{h}\right)$ in the above Lagrangian $\mathcal{L}_{h}^{\gamma}$ can be remedied by noting that $\Pi\left(\phi_{H}\right)$ is indeed an upper bound for $\Pi\left(\phi_{h}\right)$. Consequently, the final desired lower bound to the output $s$ is obtained by replacing $\Pi\left(\phi_{h}\right)$ by $\Pi\left(\phi_{H}\right)$ to give,

$$
s_{h} \geq \frac{1}{\gamma} \inf _{\varphi \in \hat{\mathcal{X}}_{H}} \mathcal{L}_{H}^{\gamma}(\varphi, \overline{\mathbf{v}}, \overline{\mathbf{q}}) \equiv s_{h}^{-}(\overline{\mathbf{v}}, \overline{\mathbf{q}}, \gamma), \quad \forall \overline{\mathbf{q}} \in \mathcal{Q}_{h}, \quad \forall \overline{\mathbf{v}} \in \mathcal{V}_{h}, \quad \forall \gamma \in \mathbb{R}^{+}
$$

where $\mathcal{L}_{H}^{\gamma}$ is now

$$
\mathcal{L}_{H}^{\gamma}(\varphi, \mathbf{v}, \mathbf{q})=\Pi(\varphi)-\Pi\left(\phi_{H}\right)+\gamma S(\varphi)+R(\mathbf{v} ; \varphi)+b(\varphi, \mathbf{q})
$$

Note that the above minimization is again carried out in the broken space $\hat{\mathcal{X}}_{h}$ and hence can be solved in an un-coupled manner on each macro-element. Once appropriate multipliers have been selected, as described in the section below, the minimizer, $\hat{\phi}_{h}^{\gamma}$, is obtained by solving the problem
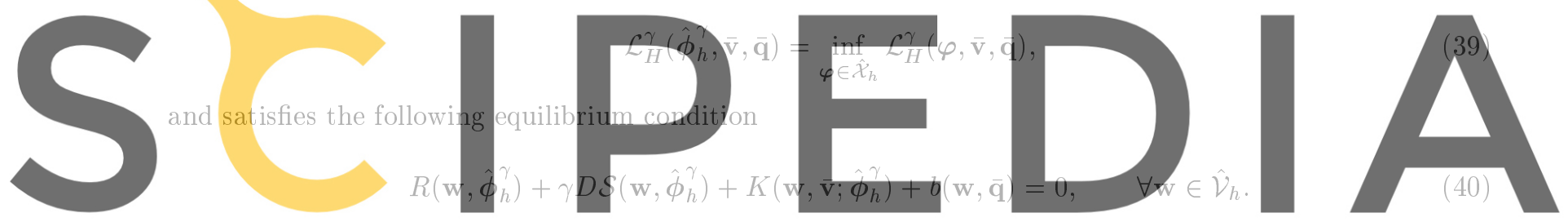

Here, $D \mathcal{S}$ denotes the first variation of the output, that is

Register for free at https//www.scipedia.com to download the version without the watermark

$$
D \mathcal{S}(\mathrm{w} ; \varphi)=\lim _{\epsilon \rightarrow 0} \frac{\mathcal{S}(\varphi+\epsilon \mathrm{W})-\mathcal{S}(\varphi)}{\epsilon} .
$$

The non-linear set of equations (40), can now be solved separately for each element with a procedure analogous to that of Appendix I.

Finally, in order to obtain an upper bound for $s_{h}$, it is only necessary to derive a lower bound $\hat{s}_{h}^{-}$for $\hat{s}=-\mathcal{S}\left(\phi_{h}\right)$, following the procedure just outlined, and then set $s_{h}^{+}=-\hat{s}_{h}^{-}[12,13,14]$. It turns out that, if we are interested in computing both upper and lower bounds, the coarse mesh adjoint can be re-used, thus gaining some computational efficiency. We also point out that, provided the minimization problem (39) is well defined and bounded infimum exists, non-linear functionals of the solution can be considered without any changes to the described procedure.

\subsection{Evaluation of the adjoint and the hybrid fluxes}

The adjoint and the hybrid fluxes are found by solving the constrained minimization problem (34) formulated in the coarse mesh spaces, $\hat{\mathcal{X}}_{H} \subset \hat{\mathcal{X}}_{h}, \mathcal{V}_{H} \subset \mathcal{V}_{h}$, and $\mathcal{Q}_{H} \subset \mathcal{Q}_{h}$. These will lead to inexpensive choices of 


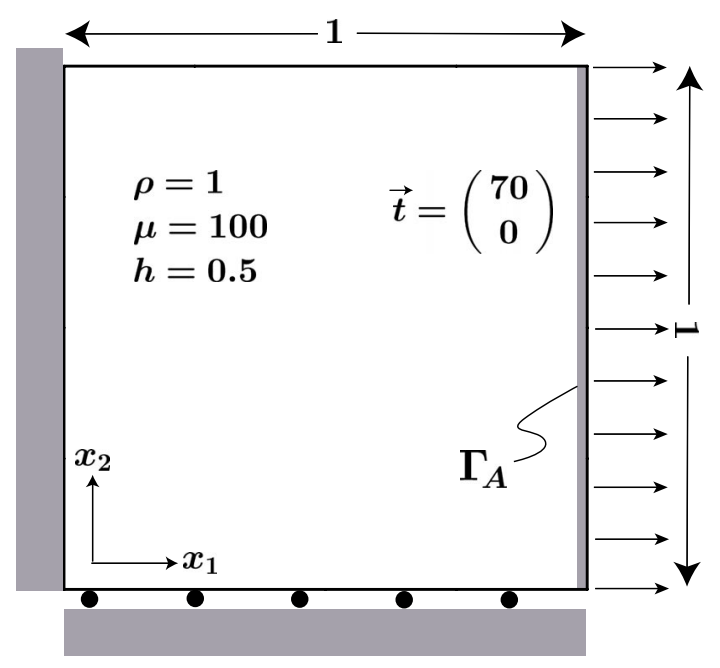

Figure 3: Square block problem

$\overline{\mathbf{q}}=\mathbf{p}_{H}$ and $\overline{\mathbf{v}}=\mathbf{u}_{H}$ which are found to yield optimum bound convergence. For this purpose, we re-write problem (34), in the coarse mesh,

$$
\gamma s_{H}=\inf _{\boldsymbol{\varphi} \in \hat{\mathcal{X}}_{H}} \sup _{\substack{\mathbf{v} \mathcal{V}_{H} \\ \mathbf{q} \in \mathcal{Q}_{H}}} \mathcal{L}_{H}^{\gamma}(\boldsymbol{\varphi}, \mathbf{v}, \mathbf{q}) .
$$

Setting the variation of the Lagrangian functional $\mathcal{L}_{H}^{\gamma}$, with respect to $\mathbf{v}$ and $\mathbf{q}$, to zero, gives

$$
\begin{aligned}
R\left(\mathbf{w} ; \phi_{H}\right)=0 & \forall \mathbf{w} \in \mathcal{V}_{H}, \\
b\left(\phi_{H}, \mathbf{q}\right)=0 & \forall \mathbf{q} \in \mathcal{Q}_{H},
\end{aligned}
$$

where, by construction, $\phi_{H}$ is the solution of the coarse grid problem (22). Considering now variations with respect to the first $\operatorname{argument} \varphi$ at $\varphi=\phi_{H}$ gives

$$
R\left(\mathbf{w} ; \phi_{H}\right)+\gamma D \mathcal{S}\left(\mathbf{w} ; \phi_{H}\right)+K\left(\mathbf{w}, \mathbf{u}_{H} ; \phi_{H}\right)+b\left(\mathbf{w}, \mathbf{p}_{H}\right)=0 \quad \forall \mathbf{w} \in \hat{\mathcal{V}}_{H} .
$$

If we first restrict this expression to variations $\mathbf{w}$ that belong to the unbroken tangent space $\mathcal{V}_{H}$, the first and last terms of the above equation vanish and a linear set of equations for the adjoint $\mathbf{u}_{H}$ emerges as,

$$
\gamma D \mathcal{S}\left(\mathbf{w} ; \phi_{H}\right)+K\left(\mathbf{w}, \mathbf{u}_{H} ; \phi_{H}\right)=0 \quad \forall \mathbf{w} \in \mathcal{V}_{H}
$$

Equation (45) can now be used to evaluate the hybrid fluxes $\mathbf{p}_{H}$ by solving an equilibration procedure analogous to that encountered in section 4.3. Finally, the free parameter $\gamma$, is chosen according to the procedure presented in Appendix II.

\section{Examples}

Two simple examples are used to demonstrate the procedures presented above: a simple extension of a square block and the deflection of a short cantilever (Cook's membrane). 


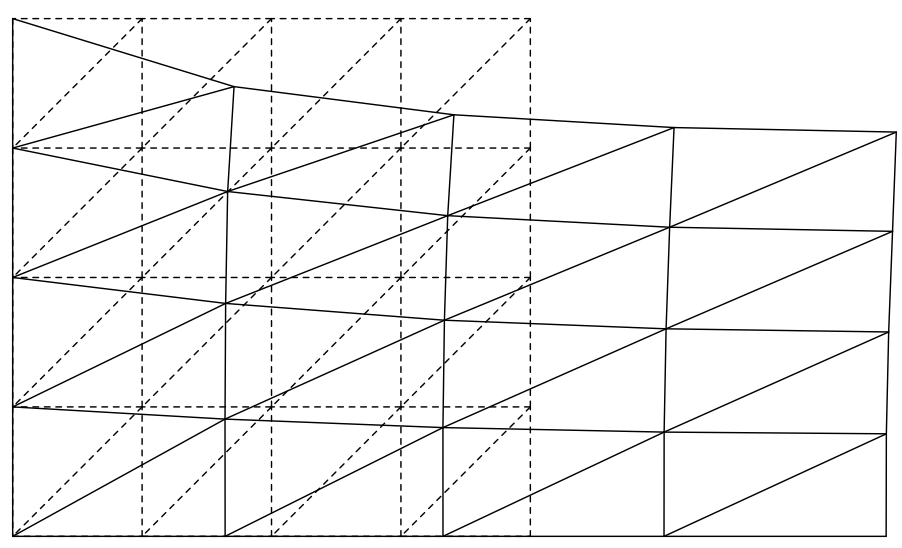

Figure 4: Square block problem - coarse mesh and deformed shape
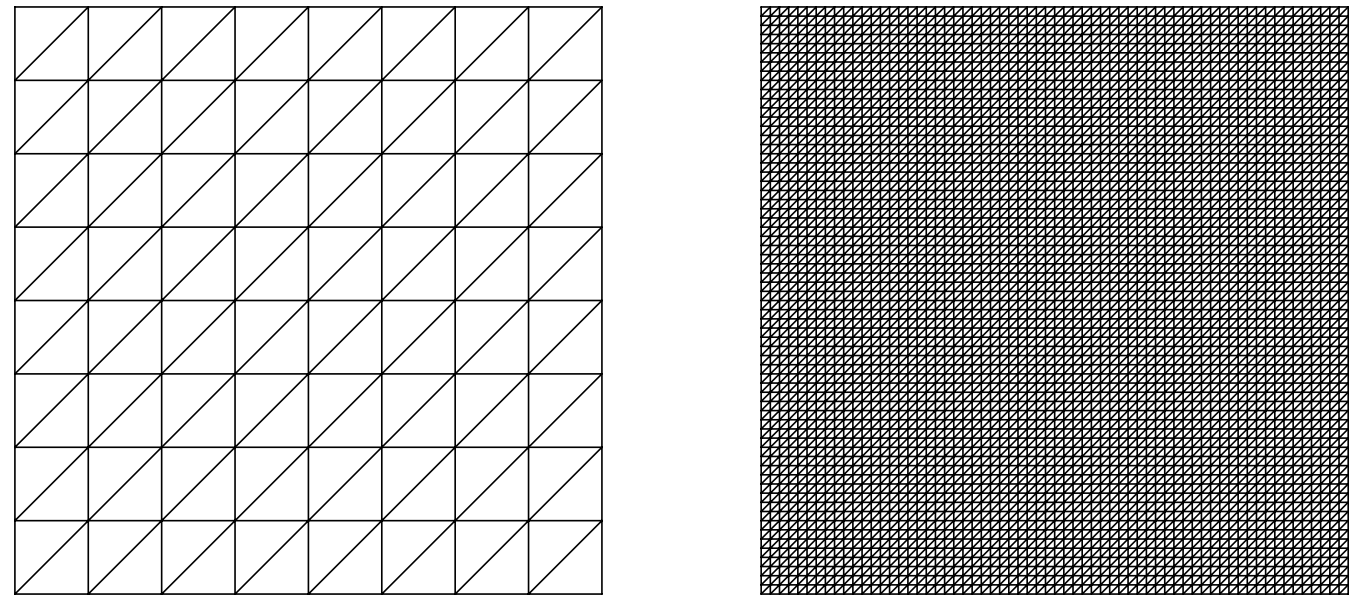

Figure 5: Square block problem - intermediate meshes $H / 2$ and $H / 8$ 


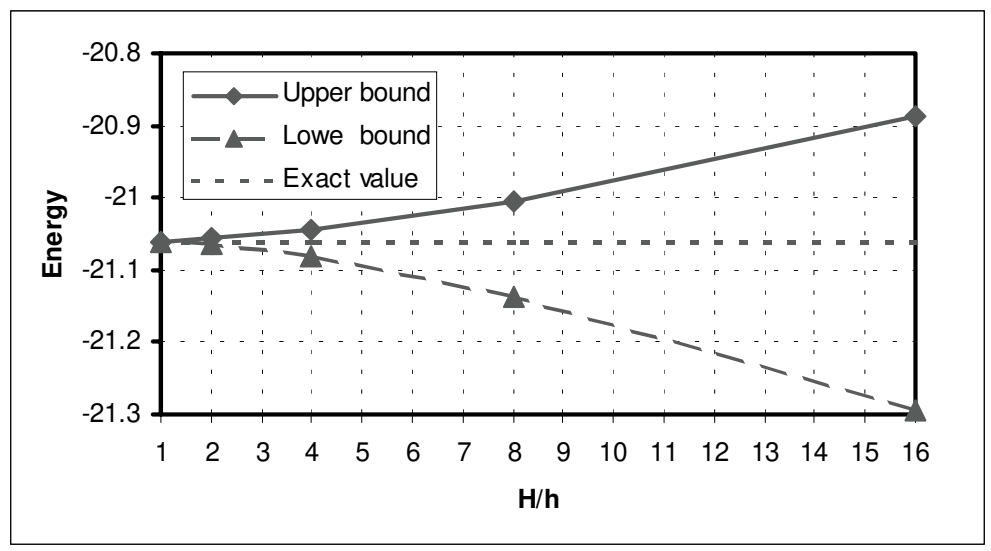

Figure 6: Square block - energy bounds

\subsection{Square block}

A square block of hyperelastic material is pulled along one side as shown in figure 3. Dimensions, boundary conditions and material properties are defined in the figure. In addition to the energy, the average displacement of the right vertical boundary of the block, will be used as the desired output for the problem.

The coarse discretization consists of 32 equal linear triangles and it is shown, together with its corresponding deformed shape, in figure 4 . The starting solution, $\phi_{H}^{0}$, to initiate the Newton-Raphson iteration (24), is taken to be simply $\phi_{H}^{0}=\mathbf{X}$. The fine mesh is obtained by subdividing each triangle into $16^{2}$ smaller elements in a regular pattern, so that $h=H / 16$. In order to check the convergence of the bounds obtained with $H$, the solution of the problem and output bounds have also been obtained using intermediate meshes with sizes $H / 2, H / 4$ and $H / 8$. Two typical intermediate meshes $H / 2$ and $H / 8$ are shown in figure 5 .

The results obtained for the energy bounds are summarized in numeric form in table 1, and graphically in figure 6. It is clear that the convergence of bound gap is quadratic with $H$.

\begin{tabular}{|c|c|c|c|}
\hline Mesh size & $\Pi_{h}^{+}$ & $\Pi_{h}^{-}$ & $\Delta_{\Pi}=\Pi_{h}^{+}-\Pi_{h}^{-}$ \\
\hline$H$ & -20.887 & -21.295 & 0.408 \\
\hline$H / 2$ & -21.004 & -21.136 & 0.132 \\
\hline$H / 4$ & -21.044 & -21.081 & 0.037 \\
\hline$H / 8$ & -21.056 & -21.063 & 0.007 \\
\hline$h \equiv H / 16$ & -21.060 & -21.060 & - \\
\hline
\end{tabular}

Table 1: Square block - Energy bounds

The bounds obtained for the average displacement are shown in table 2. The same results are illustrated graphically in figure 7 . Again the convergence of the bound gap is clearly quadratic. 


\begin{tabular}{|c|c|c|c|c|}
\hline Mesh size & $s$ & $s_{h}^{+}$ & $s_{h}^{-}$ & $\Delta_{s}=s_{h}^{+}-s_{h}^{-}$ \\
\hline$H$ & 0.6942 & 0.7196 & 0.6798 & 0.0398 \\
\hline$H / 2$ & 0.6965 & 0.7040 & 0.6924 & 0.0116 \\
\hline$H / 4$ & 0.6972 & 0.6991 & 0.6961 & 0.030 \\
\hline$H / 8$ & 0.6974 & 0.6978 & 0.6971 & 0.0007 \\
\hline$h \equiv H / 16$ & 0.6974 & 0.6974 & 0.6974 & - \\
\hline
\end{tabular}

Table 2: Square block - output bounds

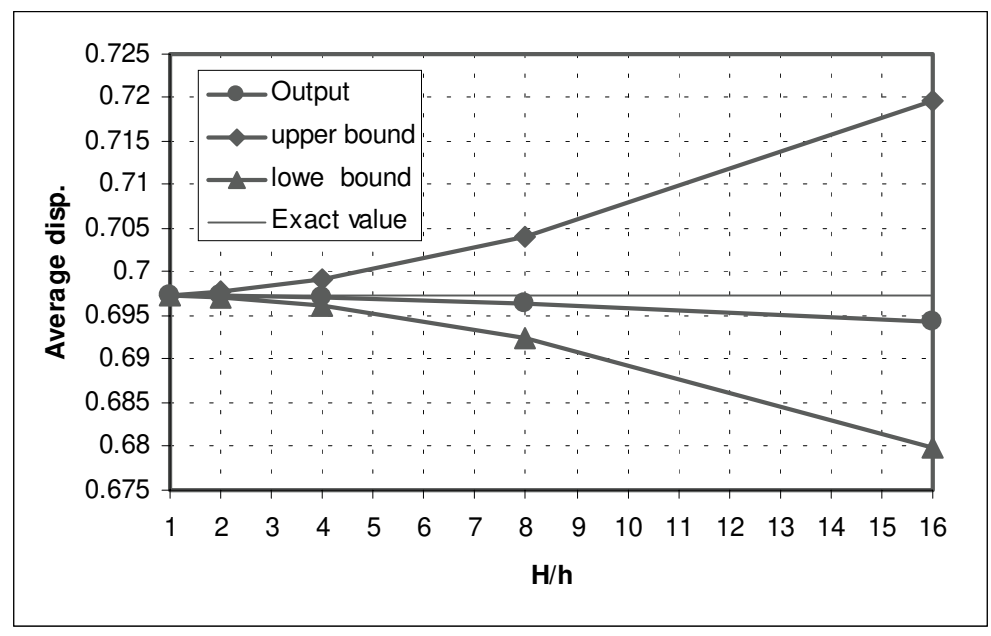

Figure 7: Square block - output bounds

\subsection{Cook's membrane}

The second example relates to the bending of a short cantilever. This is a well-known problem in solid mechanics. The geometry, loads, and boundary conditions are shown in figure 8 .

As in the previous example, a series of meshes has been used to investigate the bound convergence. These range from the coarse mesh $\mathcal{X}_{H}$, comprising 34 elements, to the fine mesh $\mathcal{X}_{h}$ with $16^{2} \times 34$ elements shown in figure 9 . In between, intermediate meshes with spacings $H / 2, H / 4$ and $H / 8$ have also been used. Figure 10 shows the final deformations obtained using the coarse mesh $\mathcal{X}_{H}$ and a finer mesh with spacing $H / 2$. In all cases, the Newton-Raphson iteration (24) used to obtain the coarse solution has been started with $\phi_{H}^{0}=\mathbf{X}$.

The upper and lower bounds obtained for the energy are listed in table 3 and depicted figure 11. We note that the lower bounds for the energy, despite still converging quadratically to the exact value when $H$ is sufficiently small, are significantly less sharp than the upper bounds, or indeed poorer than the lower bounds obtained in the previous example. The reason for the increased gap is due to the fact that many more modes of deformation are present in the broken fine mesh, which permit the local buckling of those macro-elements 


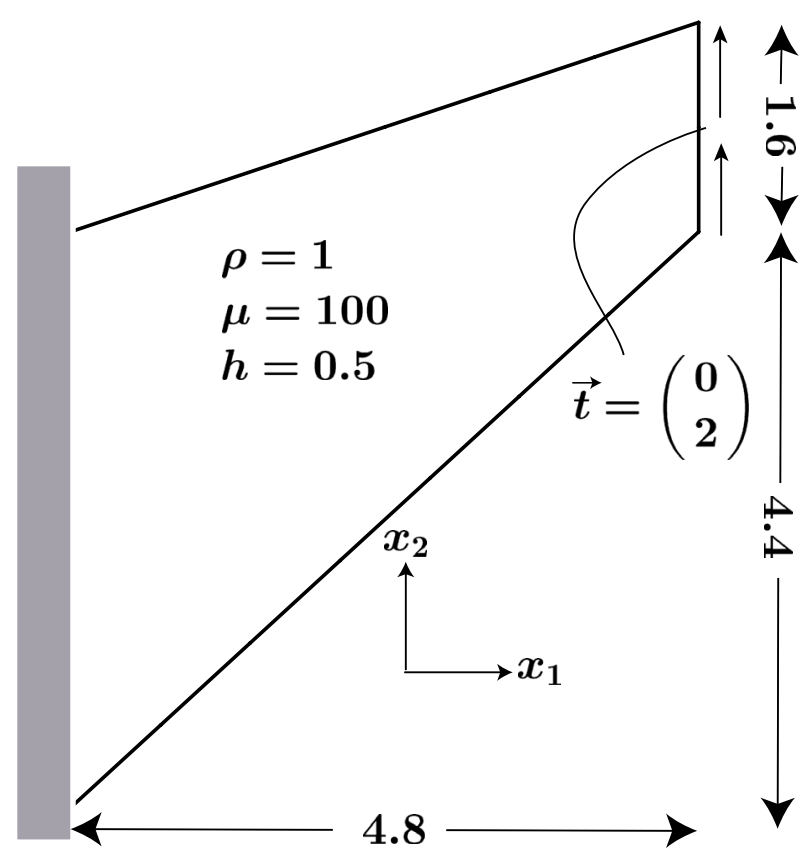

Figure 8: Cook's membrane

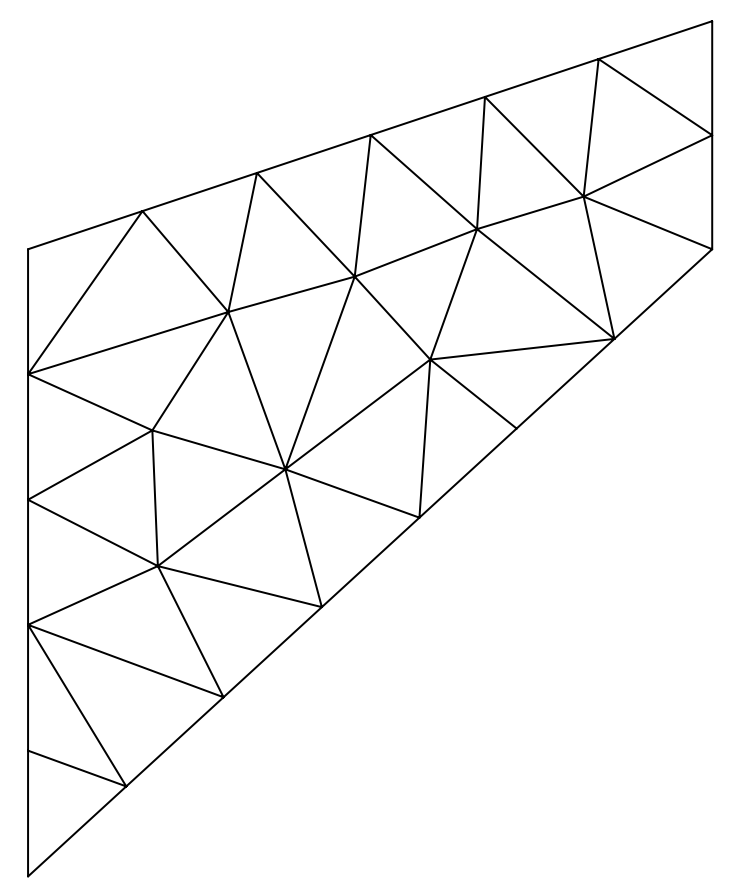

(a)

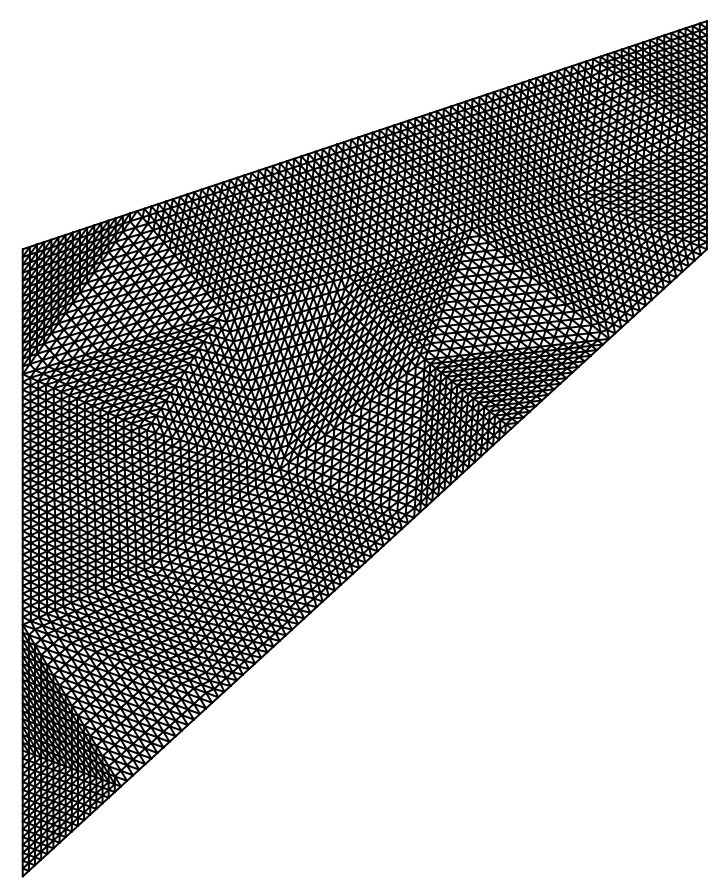

(b)

Figure 9: Cook's membrane - (a) coarse and (b) fine meshes 


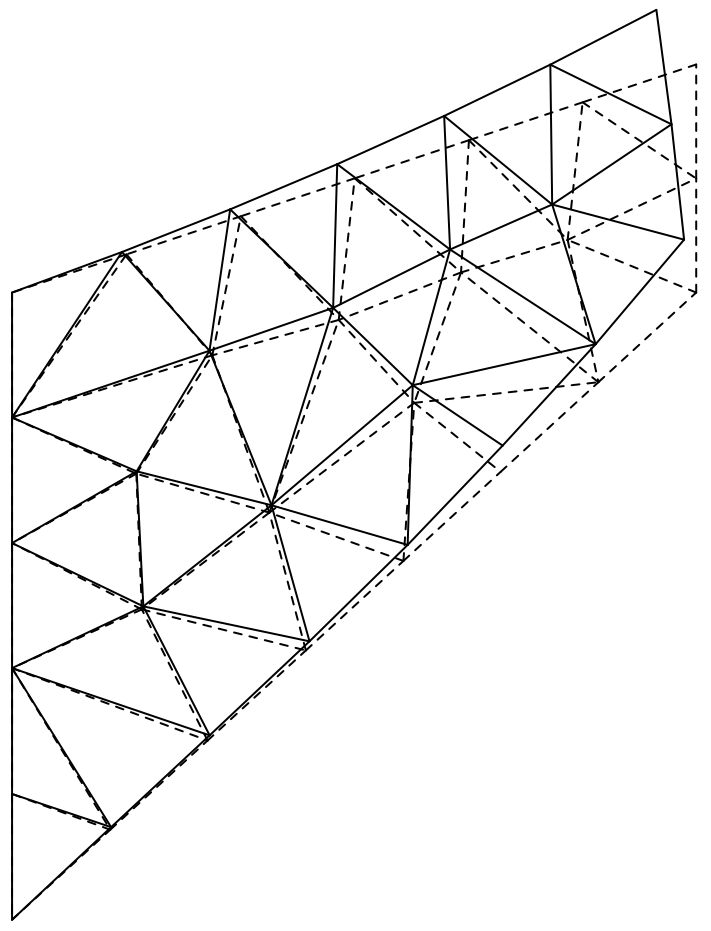

(a)

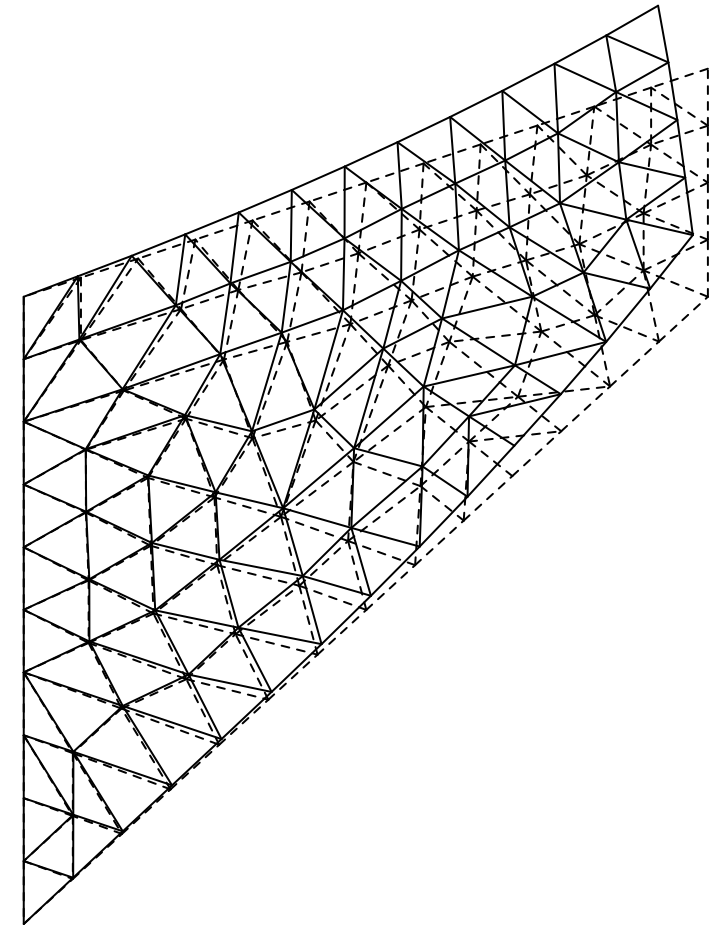

(b)

Figure 10: Cook's membrane - (a) course mesh deformation and (b) H/2 deformation

that are in compression. These local buckling modes, however, do not satisfy continuity across element edges and hence are not present in the unbroken solution. Under such conditions, the broken and unbroken solutions are qualitatively dissimilar and hence will have markedly different energy values. A clearer illustration of this problem can be seen by re-running the previous square block problem in compression rather than in tension. The deformed solutions of some local problems are shown for both the tension and compression cases in figure 12. It is clear that, for the compression case, premature local buckling is taking place well before any global buckling occurs.

\begin{tabular}{|c|c|c|c|}
\hline Mesh size & $\Pi_{h}^{+}$ & $\Pi_{h}^{-}$ & $\Delta_{\Pi}=\Pi_{h}^{+}-\Pi_{h}^{-}$ \\
\hline$H$ & -61.850 & -185.736 & 123.886 \\
\hline$H / 2$ & -72.105 & -133.850 & 61.745 \\
\hline$H / 4$ & -76.346 & -89.282 & 12.936 \\
\hline$H / 8$ & -77.832 & -79.168 & 1.336 \\
\hline$h \equiv H / 16$ & -78.010 & -78.010 & - \\
\hline
\end{tabular}

Table 3: Cook's membrane - energy output 


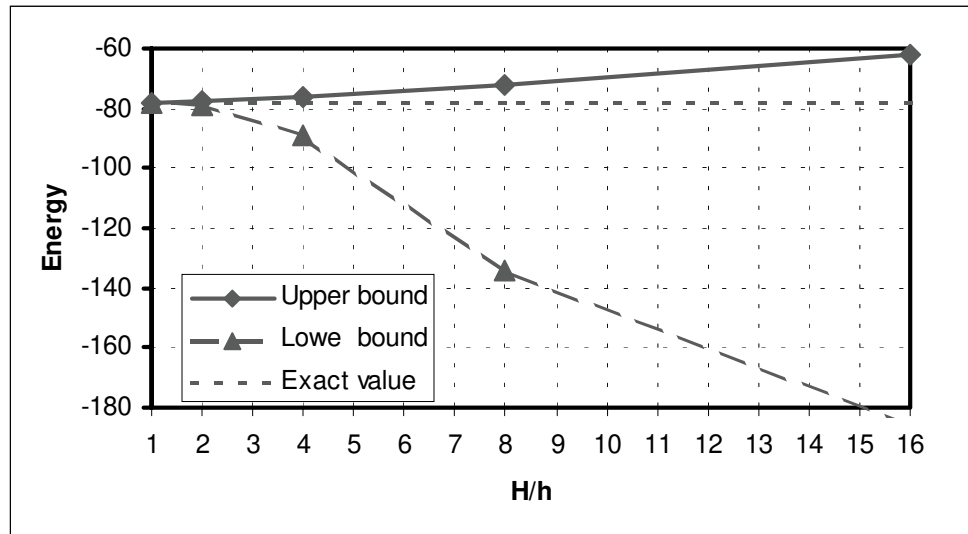

Figure 11: Cook's membrane - energy bounds

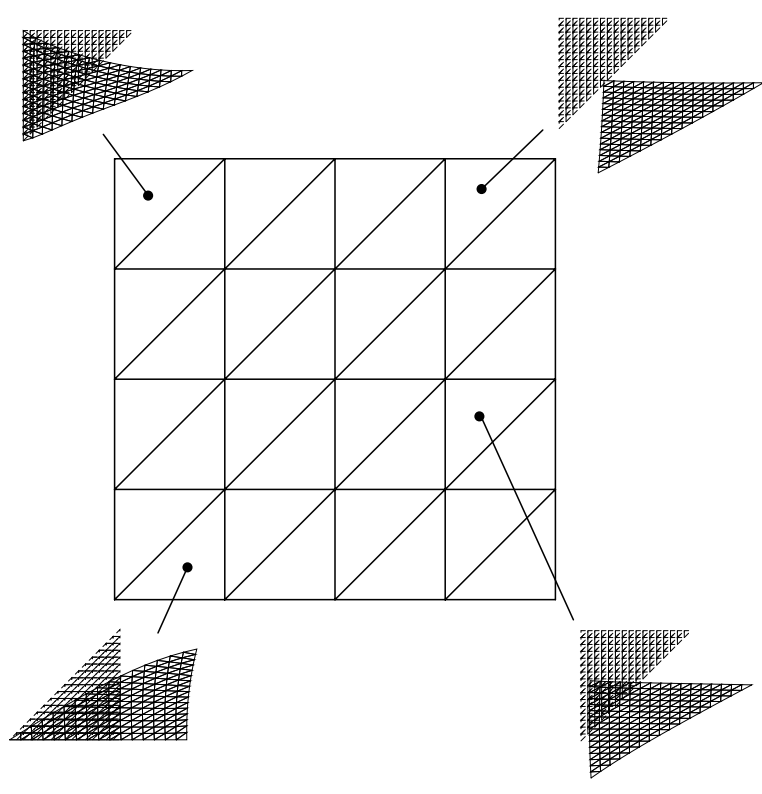

(a)

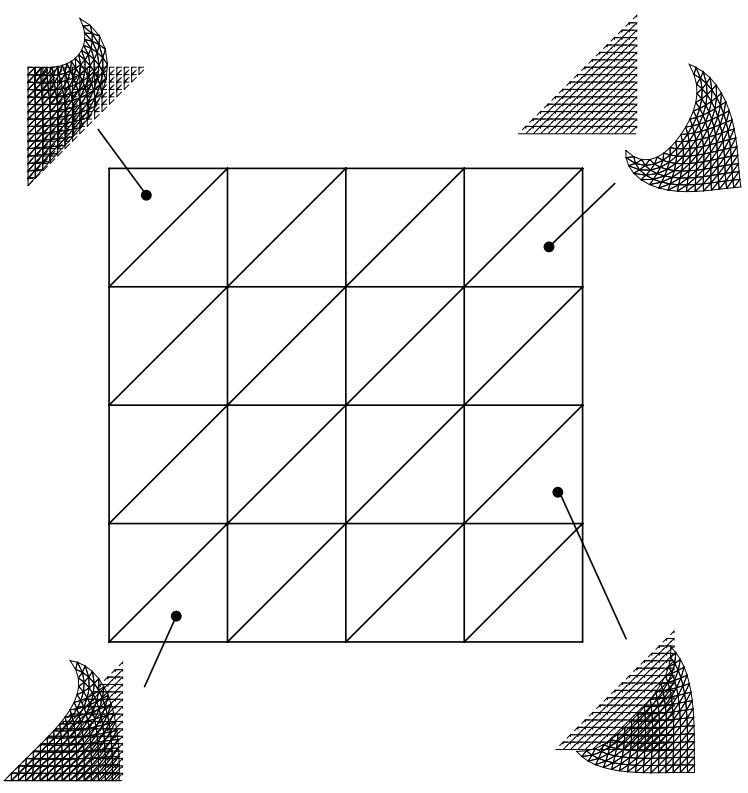

(b)

Figure 12: Local solutions for the square block in (a) tension and (b) compression 


\section{Concluding Remarks}

We have presented an efficient method for the computation of bounds for functional outputs of solutions in finite strain elasticity problems. The method has been described in detail for the simple case of two dimensional plane stress but, in principle, the procedures presented can be easily extended to three dimensions. We have only considered linear functional ouptuts, but under some restrictions more general non-linear outputs can also be dealt with.

Unlike previous error estimation procedures for non-linear problems, the approach presented is fully nonlinear and the computed bounds are uniformly valid regardless of the size of the underlying discretization. In addition, optimal bound convergence, with effectivities of order one, are obtained when the coarse grid solution is in the asymmptotic convergence range.

The method presented can be extended in a number of ways. Adaptive mesh refinement, to efficiently modify an existing discretization in order to tighten the bound gap, can be incorporated in a straightforward manner [14]. Approaches that enrich the functional spaces by increasing the polynomial order (p-methods), rather than subdividing the elements (h-methods), have been implemented successfully for linear problems, and in prinicple, could also be considered here.

Perhaps, the most severe drawback of the present approach is that of the premature buckling of the local problems. This is mostly encountered when solving problems subject to strong compression and, as previously mentioned, is due to the excessive freedom introduced by our relaxation. We are currently investigating procedures to alleviate this problem which will be the subject of a future communication.

\section{Appendix I : Solution of the local problems}

The minimization problem expressed in (30), can be carried out over each macro-element independently so that the lower bound for the energy is given by the sum of individual macro-element contributions. Thus if $\mathcal{L}_{T_{H}}$ denotes the restriction of $\mathcal{L}$ over $T_{H}$, we have

$$
\Pi_{h}^{-}\left(\mathbf{p}_{H}\right)=\sum_{T_{H} \in \mathcal{T}_{H}} \mathcal{L}_{T_{H}}\left(\left.\hat{\boldsymbol{\phi}}_{h}\right|_{T_{H}}, \mathbf{p}_{H}\right) .
$$

The local minimizers $\left.\phi_{T_{H}} \equiv \hat{\phi}_{h}\right|_{T_{H}}$ are determined by solving the local problems

$$
\mathcal{L}_{T_{H}}\left(\phi_{T_{H}}, \mathbf{p}_{H}\right)=\inf _{\varphi \in \mathcal{Z}_{h}\left(T_{H}\right)} \Pi_{T_{H}}\left(\varphi, \mathbf{t}_{T_{H}}\right) ; \quad \Pi_{T_{H}}\left(\varphi, \mathbf{t}_{T_{H}}\right) \equiv \Pi_{T_{H}}^{\mathrm{int}}(\varphi)+\Pi_{T_{H}}^{\mathrm{ext}}\left(\varphi, \mathbf{t}_{T_{H}}\right) .
$$

Here, the internal elastic strain energy potential is given by

$$
\Pi_{T_{H}}^{\mathrm{int}}(\varphi)=\int_{T_{H}} \psi(\mathbf{F}) d \Omega
$$

and the "external" energy potential, which contains the effect of the hybrid fluxes and any externally applied traction on the boundaries of $T_{H},\left.\mathbf{t}\right|_{\gamma_{H}}$, is given by

$$
\Pi_{T_{H}}^{\mathrm{ext}}\left(\boldsymbol{\varphi}, \mathbf{t}_{T_{H}}\right)=\left.\left.\sum_{\gamma_{H} \in \mathcal{E}\left(T_{H}\right)} \int_{\gamma_{H}} \mathbf{t}_{T_{H}}\right|_{\gamma_{H}} \cdot \boldsymbol{\varphi}\right|_{\gamma_{H}} H d S ;\left.\quad \mathbf{t}_{T_{H}}\right|_{\gamma_{H}}=\left.\mathbf{t}\right|_{\gamma_{H}}+\left.\sigma_{\gamma_{H}} \mathbf{p}\right|_{\gamma_{H}},
$$




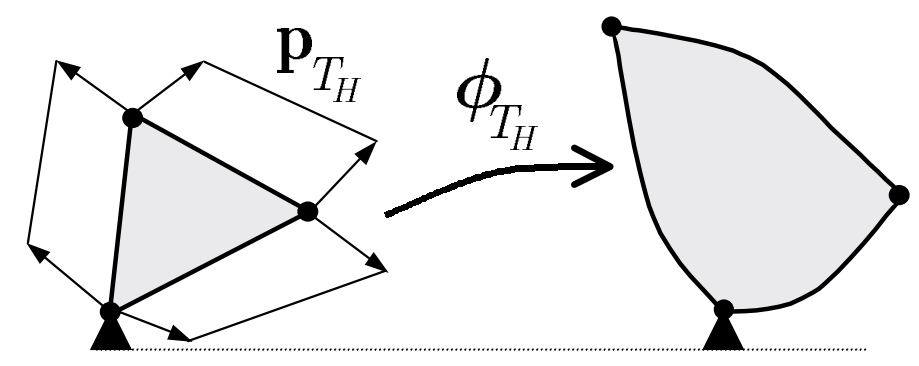

Figure 13: Local minimization problem

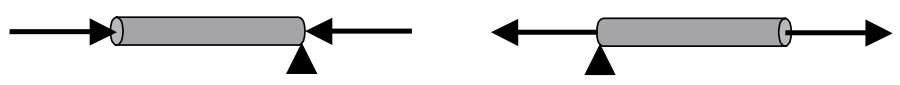

Figure 14: Illustration of solution non-uniqueness

where $\mathcal{E}\left(T_{H}\right)$, denotes the set of edges belonging to the macro-element $T_{H}$, and $\sigma_{\gamma_{H}}$ is either 1 or -1 , depending on the orientation associated to $\gamma_{H}$ (see $[2,14]$ for further details).

The stationarity conditions corresponding to problem (48) are: find $\phi_{T_{H}} \in \mathcal{Z}_{h}\left(T_{H}\right)$ such that

$$
R_{T_{H}}\left(\mathbf{v} ; \phi_{T_{H}}\right) \equiv T_{T_{H}}\left(\mathbf{v} ; \phi_{T_{H}}\right)+\Pi_{T_{H}}^{\text {ext }}\left(\mathbf{v}, \mathbf{t}_{T_{H}}\right)=0, \quad \mathbf{v} \in \mathcal{Z}_{h}\left(T_{H}\right)
$$

where $R_{T_{H}}$, denotes the local problem residual, and the internal equivalent forces $T_{T_{H}}(\mathbf{v} ; \varphi)$, are defined by the the first variation of the internal strain energy potential as

$$
T_{T_{H}}(\mathbf{v} ; \varphi)=\lim _{\epsilon \rightarrow 0} \frac{\Pi_{T_{H}}^{\mathrm{int}}(\varphi+\epsilon \mathbf{v})-\Pi_{T_{H}}^{\mathrm{int}}(\varphi)}{\epsilon} .
$$

In general, the solution of the above local problems will only be defined up to rigid body translations. The exception will be for those macro-elements with one or more edges on a Dirichlet boundary. However, provided that the external forces, $\mathbf{t}_{T_{H}}$, are in equilibrium, as guaranteed by the equilibration procedure employed to compute the hybrid fluxes, the potential to be minimized is independent of rigid body translations and hence the displacement of one point can be arbitrarily set to zero as illustrated in figure 13.

In the linear, small strain regime, rigid body rotations need to be removed from the solution space by choosing one additional suitable boundary condition. In the large strain regime, however, the external strain energy is not independent of rigid body rotations and hence the amount of rotation is solely determined by the external loads. Moreover, for any given set of self balanced external loads, one can typically find two equilibrium configurations, one in tension and one in compression, as shown in figure 14 for a simple one dimensional bar. The solution in tension represents a global minimum, whereas the solution in compression represents a minimum of the total energy with respect to all possible incremental motions except for a rigid body rotation, for which it is in fact a local maximum. This is illustrated in figure 15 , which shows the shape of the total potential energy for the simple bar problem.

It is clear that the relevant local solution will be determined by the global problem. For instance, we would expect that if the $\mathrm{H}$-macroelement corresponding to the global coarse solution is in compression, the 


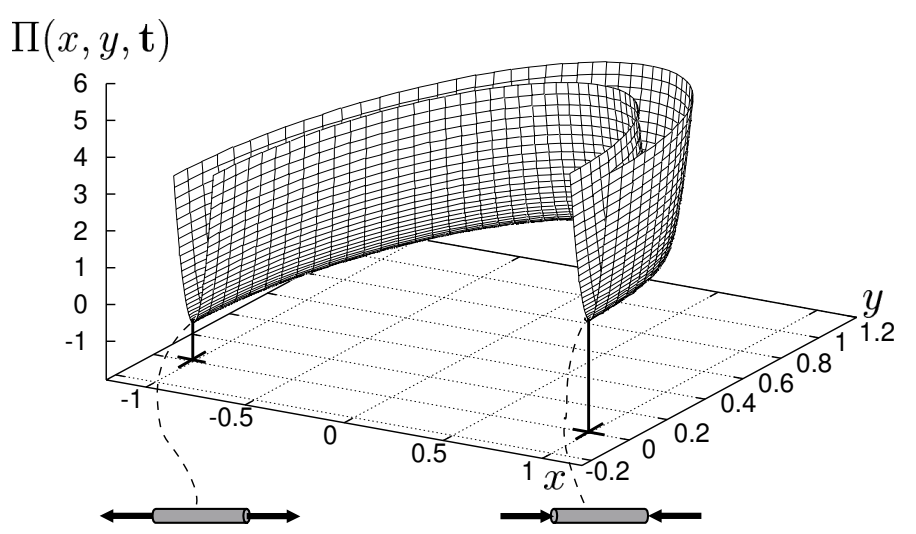

Figure 15: Total energy potential for one dimensional bar

local relevant solution would be that which is also in compression. For such case, the possible traction solution is artificially added by the relaxation of the continuity requirements in the space of local solutions.

From a practical point of view, it is found that a simple Newton-Raphson solution process does not generally converge to the desired solution in compression. In order to resolve this difficulty a simple strategy has been devised, which is effective for the two dimensional problem considered here (a similar procedure can be devised in three dimensions). In particular, the degrees of freedom that describe the space of local configurations $\mathcal{Z}_{h}\left(T_{H}\right)$ are re-defined to include explicitly a rotation angle $\vartheta$. For this purpose, a restricted space $\tilde{\mathcal{Z}}_{h}\left(T_{H}\right)$ is defined by introducing an additional support condition to remove rigid body rotations of the macro-element $T_{H}$, as:

$$
\tilde{\mathcal{Z}}_{h}\left(T_{H}\right)=\left\{\tilde{\varphi} \in \mathcal{Z}_{h}\left(T_{H}\right) \mid\left(\varphi\left(\mathbf{X}_{2}\right)-\varphi\left(\mathbf{X}_{1}\right)\right) \cdot \boldsymbol{\nu}=0\right\}
$$

where $\mathbf{X}_{1}$ and $\mathbf{X}_{2}$ are the material coordinates of the first two macro-element vertices, and $\boldsymbol{\nu}$ is a suitable fixed vector not parallel to the side joining these two nodes.

In order to recover the original solution space, one additional rotational degree of freedom $\vartheta$ is now introduced as the angle by which an arbitrary motion $\varphi$ needs to be rotated to belong to $\tilde{\mathcal{Z}}_{h}$. This rotation is illustrated in figure 16 and defines the rotation operator $\mathbf{R}_{\vartheta}$ such that $\tilde{\varphi}=\mathbf{R}_{\vartheta} \varphi$. It is clear that the total energy of the macro-element will remain unchanged if the external forces are similarly rotated, that is,

$$
\Pi_{T_{H}}\left(\boldsymbol{\varphi}, \mathbf{t}_{T_{H}}\right)=\Pi_{T_{H}}\left(\tilde{\varphi}, \mathbf{R}_{\vartheta} \mathbf{t}_{T_{H}}\right)
$$

The shape of the modified potential expressed as a function of the rotation angle and the restricted local motion is illustrated in figure 17 for the simple bar problem. It can be qualitatively observed that much of the severe non-linearity of the original problem with respect to rigid body rotations has disappeared.

Finding the stationary points with respect to both $\tilde{\varphi}$ and $\vartheta$ leads to the following set of nonlinear 


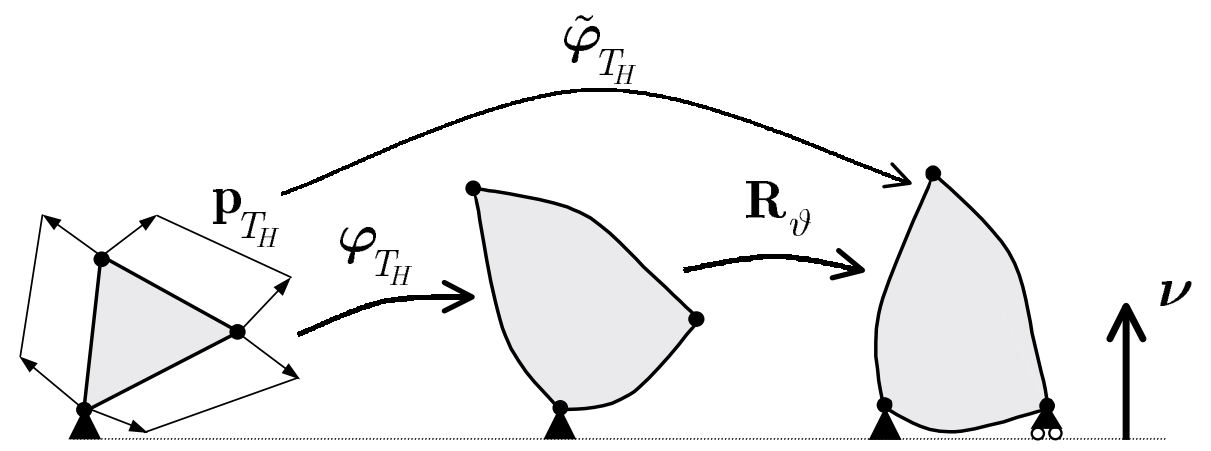

Figure 16: Modified local minimization problem

equilibrium equations for $\theta_{T_{H}}$ and $\tilde{\phi}_{T_{H}}=\mathbf{R}_{\theta_{T_{H}}} \phi_{T_{H}}$ :

$$
\begin{aligned}
T_{T_{H}}\left(\mathbf{v} ; \tilde{\boldsymbol{\phi}}_{T_{H}}\right)+\Pi_{T_{H}}^{\mathrm{ext}}\left(\mathbf{v}, \mathbf{R}_{\theta_{T_{H}}} \mathbf{t}_{T_{H}}\right) & =0 \quad \forall \mathbf{v} \in \tilde{\mathcal{Z}}_{h}\left(T_{H}\right), \\
\Pi_{T_{H}}^{\operatorname{ext}}\left(\tilde{\boldsymbol{\phi}}_{T_{H}}, \mathbf{k} \times \mathbf{R}_{\theta_{T_{H}}} \mathbf{t}_{T_{H}}\right) & =0,
\end{aligned}
$$

where $\mathbf{k}$ is the unit vector normal to the plane of motion. We note that equation (56), expresses moment equilibrium and implies that the reaction in the additional support, introduced to define $\tilde{\mathcal{Z}}_{h}\left(T_{H}\right)$, is zero.

Starting from initial guesses $\theta_{T_{H}}^{0}=0$ and $\tilde{\phi}_{T_{H}}^{0}=\left.\phi_{H}\right|_{T_{H}}$, an iterative Newton-Raphson procedure is now implemented to obtain linear equations for the increments $\Delta \tilde{\phi}=\tilde{\phi}_{T_{H}}^{i+1}-\tilde{\phi}_{T_{H}}^{i}$ and $\Delta \theta=\theta_{T_{H}}^{i+1}-\theta_{T_{H}}^{i}$ as,

$$
\begin{aligned}
K_{T_{H}}\left(\mathbf{v}, \Delta \tilde{\boldsymbol{\phi}} ; \tilde{\phi}_{T_{H}}^{i}\right)+\Pi_{T_{H}}^{\mathrm{ext}}\left(\mathbf{v}, \Delta \theta \mathbf{k} \times \mathbf{R}_{\theta_{T_{H}^{i}}^{i}} \mathbf{t}_{T_{H}}\right) & =-R_{T_{H}}\left(\mathbf{v} ; \tilde{\boldsymbol{\phi}}_{T_{H}}^{i}\right) \quad \forall \mathbf{v} \in \tilde{\mathcal{Z}}_{h}\left(T_{H}\right), \\
\Pi_{T_{H}}^{\mathrm{ext}}\left(\Delta \tilde{\boldsymbol{\phi}}, \mathbf{k} \times \mathbf{R}_{\theta_{T_{H}}^{i}} \mathbf{t}_{T_{H}}\right)-\Pi_{T_{H}}^{\mathrm{ext}}\left(\tilde{\phi}_{T_{H}}^{i}, \Delta \theta \mathbf{R}_{\theta_{T_{H}}^{i}} \mathbf{t}_{T_{H}}\right) & =-\Pi_{T_{H}}^{\mathrm{ext}}\left(\tilde{\boldsymbol{\phi}}_{T_{H}}^{i}, \mathbf{k} \times \mathbf{R}_{\theta_{T_{H}}^{i}} \mathbf{t}_{T_{H}}\right) .
\end{aligned}
$$

The solution of the above linear system can be facilitated by decomposing $\Delta \tilde{\phi}$ as,

$$
\Delta \tilde{\phi}=\Delta \tilde{\phi}_{R}+\Delta \theta \Delta \tilde{\phi}_{\theta},
$$

where,

$$
\begin{aligned}
K_{T_{H}}\left(\mathbf{v}, \Delta \tilde{\boldsymbol{\phi}}_{R} ; \tilde{\boldsymbol{\phi}}_{T_{H}}^{i}\right) & =-R_{T_{H}}\left(\mathbf{v} ; \tilde{\boldsymbol{\phi}}_{T_{H}}^{i}\right) \quad \forall \mathbf{v} \in \tilde{\mathcal{Z}}_{h}\left(T_{H}\right), \\
K_{T_{H}}\left(\mathbf{v}, \Delta \tilde{\boldsymbol{\phi}}_{\theta} ; \tilde{\boldsymbol{\phi}}_{T_{H}}^{i}\right) & =-\Pi_{T_{H}}^{\mathrm{ext}}\left(\mathbf{v}, \mathbf{k} \times \mathbf{R}_{\theta_{T_{H}^{i}}^{i}} \mathbf{t}_{T_{H}}\right) \quad \forall \mathbf{v} \in \tilde{\mathcal{Z}}_{h}\left(T_{H}\right) .
\end{aligned}
$$

Substituting the decomposition (59) into equation (58) gives the angle increment after simple algebra as,

$$
\Delta \theta=-\frac{\Pi_{T_{H}}^{\mathrm{ext}}\left(\tilde{\boldsymbol{\phi}}_{T_{H}}^{i}+\Delta \tilde{\boldsymbol{\phi}}_{R}, \mathbf{k} \times \mathbf{R}_{\theta_{T_{H}}^{i}} \mathbf{t}_{T_{H}}\right)}{\Pi_{T_{H}}^{\mathrm{ext}}\left(\Delta \tilde{\boldsymbol{\phi}}_{\theta}, \mathbf{k} \times \mathbf{R}_{\theta_{T_{H}}^{i}} \mathbf{t}_{T_{H}}\right)-\Pi_{T_{H}}^{\mathrm{ext}}\left(\tilde{\boldsymbol{\phi}}_{T_{H}}^{i}, \mathbf{R}_{\theta_{T_{H}}^{i}} \mathbf{t}_{T_{H}}\right)} .
$$

The above angle increment can now be substituted back into equation (59) to give the iterative increment $\Delta \tilde{\phi}$ in $\phi_{T_{H}}$.

Finally, we set

$$
\Pi_{T_{H}}\left(\phi_{T_{H}}, \mathbf{t}_{T_{H}}\right)=\Pi_{T_{H}}\left(\tilde{\phi}_{T_{H}}, \mathbf{R}_{\theta_{T_{H}}} \mathbf{t}_{T_{H}}\right) .
$$

We have found that the above artifice proves effective to give the appropriate local solution in the presence of moderate compression. 


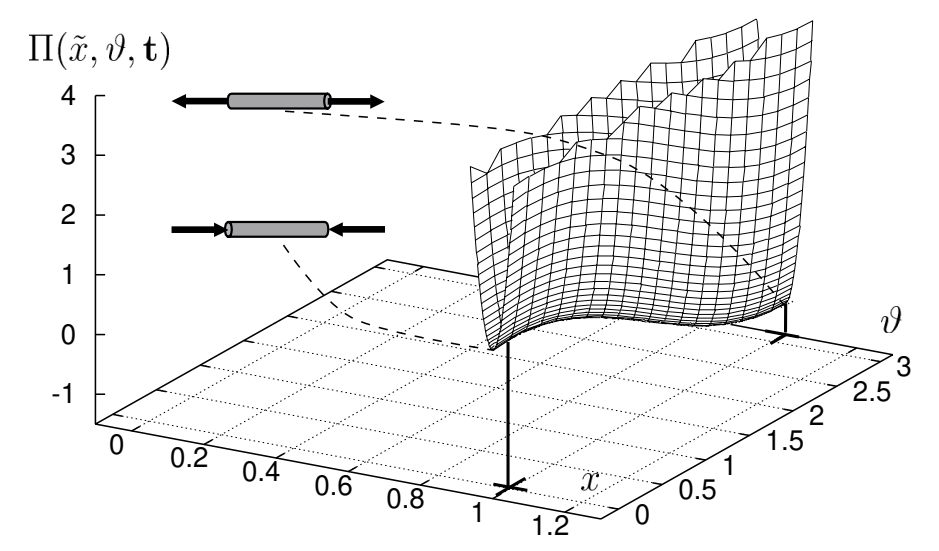

Figure 17: Total energy potential for one dimensional bar in transformed solution space

\section{Appendix II : Evaluation of the parameter $\gamma$}

The parameter $\gamma$ was introduced in equation (38) as a device to harmonize the physical units in the Lagrangian functional. This has the inevitable consequence of making output bound $s_{h}^{-}$depend on the chosen value for this factor. Ideally, one should find the value of $\gamma$ which leads to the highest lower bound. In fact, it is possible to solve for $\gamma$ as an additional unknown by maximizing the expression for $s_{h}^{-}$with respect to $\gamma$, thus obtaining one additional equation that permits the evaluation of the optimum $\gamma$. This equation, however, is highly nonlinear and cumbersome to solve, as it links the solution of the local problems.

A much simpler alternative is obtained by assuming small values of $\gamma$ and taking a truncated Taylor series expansion of the potential $\mathcal{L}_{H}$ about the point $\gamma=0$, which coincides with the case discussed in section (6), where the bounds for the total potential energy where evaluated. For this purpose, it is first useful to re-write equation (37) as,

$$
s_{h}^{-}\left(\mathbf{u}_{H}, \mathbf{p}_{H}, \gamma\right)=\frac{f(\gamma)}{\gamma}, \quad f(\gamma)=\Pi\left(\hat{\phi}_{h}^{\gamma}\right)-\Pi\left(\phi_{H}\right)+\gamma \mathcal{S}\left(\hat{\boldsymbol{\phi}}_{h}^{\gamma}\right)+R\left(\mathbf{u}_{H} ; \hat{\boldsymbol{\phi}}_{h}^{\gamma}\right)+b\left(\hat{\boldsymbol{\phi}}_{h}^{\gamma}, \mathbf{p}_{H}\right) .
$$

Using a truncated Taylor series expansion of $f(\gamma)$ about $\gamma=0$, taking linear and quadratic terms, an approximate optimum value of $\gamma$ is easily found as,

$$
\gamma_{\mathrm{opt}}=\sqrt{\frac{2 f(0)}{f^{\prime \prime}(0)}} .
$$

The value of $f(0)$ is simply,

$$
f(0)=\Pi\left(\hat{\phi}_{h}^{0}\right)-\Pi\left(\phi_{H}\right)+b\left(\hat{\phi}_{h}^{0}, \mathbf{p}_{H}^{0}\right) .
$$

where $\hat{\boldsymbol{\phi}}_{h}^{0}$ and $\mathbf{p}_{H}^{0}$ coincide with the local solution $\hat{\boldsymbol{\phi}}_{h}$ and hybrid fluxes $\mathbf{p}_{H}$ obtained in section (6).

The first and second derivatives of $f(\gamma)$, are readily evaluated from equation (64) and with the help of 
equilibrium statement (40) to give,

$$
\begin{aligned}
f^{\prime}(\gamma) & =\mathcal{S}\left(\hat{\boldsymbol{\phi}}_{h}^{\gamma}\right)+R\left(\frac{d \mathbf{u}_{H}}{d \gamma} ; \hat{\boldsymbol{\phi}}_{h}^{\gamma}\right)+b\left(\hat{\boldsymbol{\phi}}_{h}^{\gamma}, \frac{d \mathbf{p}_{H}}{d \gamma}\right) \\
f^{\prime \prime}(\gamma) & =D \mathcal{S}\left(\frac{d \hat{\boldsymbol{\phi}}_{h}}{d \gamma} ; \hat{\boldsymbol{\phi}}_{h}^{\gamma}\right)+K\left(\frac{d \mathbf{u}_{H}}{d \gamma}, \frac{d \hat{\boldsymbol{\phi}}_{h}^{\gamma}}{d \gamma} ; \hat{\boldsymbol{\phi}}_{h}^{\gamma}\right)+b\left(\frac{d \hat{\boldsymbol{\phi}}_{h}^{\gamma}}{d \gamma}, \frac{d \mathbf{p}_{H}}{d \gamma}\right),
\end{aligned}
$$

where the derivatives $d \mathbf{u}_{H} / d \gamma$ and $d \mathbf{p}_{H} / d \gamma$ are obtained by differentiating with respect to $\gamma$ equations (46) and (45) respectively, to give,

$$
\begin{array}{rlrl}
-D \mathcal{S}\left(\mathbf{w} ; \phi_{H}\right)+K\left(\mathbf{w}, \frac{d \mathbf{u}_{H}}{d \gamma} ; \phi_{H}\right) & =0 & & \forall \mathbf{w} \in \mathcal{V}_{H}, \\
-D \mathcal{S}\left(\mathbf{w} ; \phi_{H}\right)+K\left(\mathbf{w}, \frac{d \mathbf{u}_{H}}{d \gamma} ; \phi_{H}\right)+b\left(\mathbf{w}, \frac{d \mathbf{p}_{H}}{d \gamma}\right)= & 0 & \forall \mathbf{w} \in \hat{\mathcal{V}}_{H} .
\end{array}
$$

Similarly, the derivative of $\hat{\phi}_{h}^{\gamma}$ with respect to $\gamma$ can be evaluated by differentiating equation (40), which at $\gamma=0$ yields,

$$
K\left(\mathbf{w}, \frac{d \hat{\boldsymbol{\phi}}_{h}^{\gamma}}{d \gamma} ; \hat{\boldsymbol{\phi}}_{h}^{0}\right)+D \mathcal{S}\left(\mathbf{w}, \hat{\boldsymbol{\phi}}_{h}^{0}\right)+K\left(\mathbf{w}, \frac{d \mathbf{u}_{H}}{d \gamma} ; \hat{\phi}_{h}^{0}\right)+b\left(\mathbf{w}, \frac{d \mathbf{p}_{H}}{d \gamma}\right)=0 \quad \forall \mathbf{w} \in \hat{\mathcal{V}}_{h} .
$$

Finally, note that this equation enables the expression for $f^{\prime \prime}(0)$ from equation (70) to be simplified to,

$$
f^{\prime \prime}(0)=-K\left(\frac{d \hat{\phi}_{h}}{d \gamma}, \frac{d \hat{\phi}_{h}}{d \gamma} ; \hat{\phi}_{h}^{0}\right)
$$

The above procedure provides a simple mechanism for evaluating a reasonable value of $\gamma$. It must be emphasized, however, that the lower and upper bounds of $s$ are, not only valid for any value of $\gamma$, but also the bound gap converges quadratically to zero regardless of $\gamma$.

\section{Acknowledgements}

This work was partially carried out while J. Peraire was on sabbatical leave at the Universitat Politecnica de Catalunya, Barcelona. The authors would like to acknowledge the support provided by the Ministry of Spanish Education, the Singapore-MIT Alliance and Sandia National Laboratories for supporting the work reported here. The authors would also like to acknowledge fruitful discussions and collaboration with Professors A.T. Patera from MIT, and Y. Maday from Paris VI.

\section{References}

[1] M. Ainsworth and T.J. Oden, A unified approach to a posteriori error estimation based on element residula methods, Numer. Math., 65:23-50, 1993.

[2] M. Ainsworth and J.T. Oden, A Posteriori Error Estimation in Finite Element Analysis, WileyInterscience, 2000, 
[3] I. Babuška and W.C. Rheinboltd, A posteriori error estimates for the finite element method, Int. J. Num. Meth. in Engrg., 12:1597-1615, 1978.

[4] I. Babuška, T. Strouboulis, C.S. Upadhyay, and S.K. Ganaraj, A posteriori estimation and adaptive-control of the pollution error in the h-version of the finite-element method, Int. J. Num. Meth. in Engrg., 38(24):4207-4235, 1995.

[5] R. Bank and A. Weiser, Some a posteriori error estimators for elliptic partial differential equations, Math. Comp., 44:283-301, 1985.

[6] R. Becker and R. Rannacher, A feed-back approach to error control in finite element methods: basic analysis and examples, East-West J. Numer. Math., 4:237-264, 1996.

[7] J. Bonet and R.D. Wood, Nonlinear continuum mechanics for finite element analysis, Cambridge University Press, 1997.

[8] F. Brezzi and M. Fortin, Mixed and hybrid finite element methods, Springer-Verlag, New York, 1991.

[9] P. Ladevèze and D. Leguillon, Error estimate procedure in the finite element method and applications, SIAM J. Numer. Anal., 20:485-509, 1983.

[10] F. Larsson, P. Hansbo and K. Runesson, Strategies for computing goal-oriented a posteriori error measures in nonlinear elasticity, submitted to Int. J. Num. Meth. in Engrg., 2001

[11] J.E. Marsden and T.J.R. Hughes, Mathematical foundations of elasticity, Prentice-Hall, 1983.

[12] M. Paraschivoiu and A.T. Patera, A hierarchy duality approach to bounds for the outputs of partial differential equations, Comput. Methods Appl. Mech. Engrg., 158:389-407 1998.

[13] M. Paraschivoiu, J. Peraire and A.T. Patera, A posteriori finite element bounds for linear-functional outputs of elliptic partial differential equations, Comput. Methods Appl. Mech. Engrg., 150:289-312 1997.

[14] J. Peraire and A.T. Patera, Bounds for linear-functional outputs of coercive partial differential equations: local indicators and adpative refinement, in Advances in Adaptive Computational Methods in Mechanics, P. Ladevèze and J.T. Oden editors. Elsevier, 1998.

[15] A. Prudhomme and J.T. Oden, On goal-oriented error estimation for elliptic problems: Application to control of pointwise errors, Comput. Methods Appl. Mech. Engrg., 176(14):313-331, 1999.

[16] R. Rannacher and F.T. Suttmeier, A feed-back approach to error control in finite element methods: Application to linear elasticity, Comput. Mech., 19(5):434-446, 1997. 
[17] R. Rannacher and F.T. Suttmeier, A posteriori error estimation and mesh adaption for finite element models in elasto-plasticity, Comput. Methods Appl. Mech. Engrg., 176(14):333-361, 1999.

[18] G. Strang, Introduction to applied mathematics, Wellesley-Cambridge Press, 1986. 